1

3 Vidya Mangala Prasad ${ }^{1}$, Daniel P. Leaman ${ }^{4}$, Klaus N. Lovendahl ${ }^{1}$, Mark A. Benhaim ${ }^{1} \uparrow$, Edgar

\section{*Correspondence to: kklee@uw.edu}

†Current Address: Alkermes, Inc., 900 Winter St, Waltham, MA 02451, USA.

\title{
16 Summary:
}

17 HIV-1 Env mediates viral entry into host cells and is the sole target for neutralizing antibodies.

18 However, Env structure and organization in its native virion context has eluded detailed

19 characterization. Here we used cryo-electron tomography to analyze Env in mature and immature

20 HIV-1 particles. Immature particles showed distinct Env positioning relative to the underlying Gag

21 lattice, providing insights into long-standing questions about Env incorporation. A 9.1A sub-

22 tomogram averaged reconstruction of virion-bound Env in conjunction with structural mass

23 spectrometry revealed unexpected features, including a variable central core of the gp41 subunit, 
24 heterogeneous glycosylation between protomers plus a flexible stalk that allows Env tilting and

25 variable exposure of neutralizing epitopes. Together, our results provide an integrative

26 understanding of HIV assembly and structural variation in Env antigen presentation.

28 Keywords: HIV Env glycoprotein, HIV assembly, Gag-Env interaction, Cryo-electron

29 tomography, sub-tomogram averaging, structural mass spectroscopy.

\section{Introduction:}

Human Immunodeficiency Virus-1 (HIV-1) continues to infect nearly two million people

33 worldwide each year, with no available vaccine against the virus (Pandey and Galvani 2019). The

34 envelope glycoprotein (Env) on the surface of HIV-1 is an essential viral entry machine that

35 mediates binding to host cell receptors and subsequent membrane fusion. As the sole target for

36 neutralizing antibodies, Env is also of singular importance for vaccine design efforts (van Gils and

37 Sanders 2013). Env is translated as a precursor gp160 protein, which trimerizes, and is cleaved

38 into gp120 and gp41 subunits that are primarily responsible for receptor binding and fusion,

39 respectively. The gp120 and gp41 subunits remain as non-covalently associated heterodimers with

40 gp41 embedded in the viral membrane via its transmembrane domain (TMD) and the C-terminal

41 cytoplasmic domain (CTD) in the viral lumen. On incorporation into budding virions, Env CTD

42 interacts with the matrix domain (MA) of immature Gag polyprotein which assembles as a

43 membrane-associated lattice on the inner side of the viral bilayer (Checkley, Luttge et al. 2011,

44 Tedbury and Freed 2014).

45 Recent insights into Env structure have primarily come from studies of engineered,

46 truncated forms of the trimer ectodomain (such as SOSIPs) (Ward and Wilson 2015, Stewart- 
47 Jones, Soto et al. 2016), while details of Env structure in its viral membrane context, including its

48 organization relative to other viral components, has remained elusive. In order to understand how

49 Env is assembled on virions and how it differs structurally from soluble trimers, we carried out

50 cryo-electron tomography (cryo-ET) with sub-tomogram averaging, and structural mass

51 spectrometry analysis of native Env displayed on immature and mature Gag-bearing HIV-1 virus-

52 like particles. These studies advance our knowledge of native Env via insights gained from cryo-

53 ET of intact viral particles.

\section{Results and Discussion:}

Virion particles bearing ADA.CM Env, a variant of subtype B isolate ADA that was

57 selected for stability (Leaman and Zwick 2013), were produced in human HEK 293T cells as

58 previously described (Stano, Leaman et al. 2017) (Figure 1A, B). These membrane enveloped

59 virus-like particles, referred to as high Env (h)VLPs, display elevated levels of Env trimers that

60 are fully functional for mediating receptor binding and entry. Env on hVLPs retain the antigenic

61 profile of full-length Env (Figure S1 and Table S1), despite a C-terminal truncation of 102 amino

62 acids in CTD (Stano, Leaman et al. 2017). hVLPs assemble around a functional Gag layer that

63 undergoes proteolytic maturation and thus, have the composition of authentic HIV-1 virions, but

64 are replication incompetent because the packaged genome lacks the Env gene (Leaman and Zwick

65 2013, Stano, Leaman et al. 2017). Purified hVLPs were treated with aldrithiol-2 (AT-2), which

66 interrupts nucleocapsid interaction with RNA, to ensure non-infectivity (Rossio, Esser et al. 1998).

67 Antibody binding experiments showed that the antigenicity of hVLP-Env was unaffected by AT-

682 treatment (Figure S2), suggesting the hVLP-Env trimer conformation is unaltered by AT-2,

69 which is in contrast to a recent report for a neutralization sensitive HIV Env (Li, Li et al. 2020). 
70 The high density of surface Env on hVLPs allowed us to gather sufficient cryo-ET data to obtain

71 a $9.1 \AA$ resolution structure of native Env in its authentic context on virus-like particles (Figure 1C

72 and S3).

73

74 Env structure from immature particles reveal interactions with Gag

75 Though the hVLPs in our sample population were predominantly mature, we also observed

76 particles at different stages of maturation (Figure 1D). Approximately $3 \%$ of the particles were

77 fully immature, exhibiting a clear, assembled Gag lattice beneath the viral membrane (Figure 1A,

78 D). Env sub-tomogram volumes from only immature particles were averaged together to give

79 density maps at $\sim 31 \AA$ (C3 symmetry) and $\sim 34 \AA$ (C1 symmetry) (Figure $2 \mathrm{~A}$ and S4). The Env

80 structure from immature particles closely resembles the sub-nanometer Env structure generated

81 from the total hVLP population (Figure 1C) and other reported Env ectodomain structures (Kwon,

82 Pancera et al. 2015, Ward and Wilson 2015). In the averaged immature Env map, a third density

83 layer is seen underneath the membrane bilayer corresponding to the Gag lattice position (Figure

84 1A, 2A, B and Figure S4B, C). Focused refinement of this internal third layer revealed a distinct

85 structure that clearly resembles a lattice formed by the capsid domain (CA) of immature Gag

86 polyprotein (Figure 2B and Figure S4D) (Schur, Hagen et al. 2015). Thus, structures of trimeric

87 Env ectodomain (Pan, Peng et al. 2020) and hexameric Gag-CA (Schur, Hagen et al. 2015) were

88 fitted as rigid bodies into corresponding densities in the averaged map (Figure 2C). No clear

89 density for the Env TMD-CTD was observed in the averaged structure, even though the TMD is

90 seen in individual raw tomograms (Figure 2C), suggesting that the TMD is not strictly aligned

91 with the ectodomain or Gag-CA. The structure of the TMD-CTD (PDB ID: 6UJV) was thus placed

92 into the map based on its position relative to the Env ectodomain (Figure 2C) (Piai, Fu et al. 2020). 
The N-terminal MA domain of immature Gag polyprotein assembles along the inner

94 membrane leaflet as a hexameric lattice of trimers (Frank, Narayan et al. 2015, Tedbury, Novikova

95 et al. 2016) and is connected by a flexible linker to the Gag-CA lattice underneath (Tedbury and

96 Freed 2014). Although regularly spaced puncta corresponding to the Gag-MA domain can be

97 discerned directly underneath the inner leaflet and Env ectodomains in immature hVLP tomograms

98 (Figure 2C), distinguishable density for the Gag-MA layer was not seen in the averaged immature

99 map implying that the Gag-MA lattice (Tedbury and Freed 2014, Frank, Narayan et al. 2015,

100 Tedbury, Novikova et al. 2016), may have subtle variations in arrangement that deviate from Gag-

101 CA or Env symmetry axes. Hence, based on the expected position of Gag-MA relative to the Gag-

102 CA lattice (Frank, Narayan et al. 2015, Tedbury, Novikova et al. 2016), the MA lattice structure

103 was placed into the map (Figure 2C). As a result, juxtaposition of Env CTD and Gag-MA occurs

104 right at the bottom of the inner membrane as would be expected from the raw tomograms (Figure

105 2C). From this composite model, the relative position of Env CTD, when viewed normal to the

106 membrane, is on the rim of the hexameric Gag-CA lattice along its 2-fold axis (Fig2D). This

107 observation can also be corroborated in the raw tomograms (Figure 2E). Thus, the trimeric Env

108 CTD does not lie in the central hole formed by the immature Gag lattice as has been hypothesized

109 (Tedbury and Freed 2014, Tedbury, Novikova et al. 2016), but instead is positioned on its

110 hexameric rim (Figure 2D, F). In this configuration, the modeled Env CTD makes direct contact

111 with critical residues on MA previously shown to be necessary for Env incorporation in virions

112 (Figure 2F) (Tedbury and Freed 2014, Tedbury, Novikova et al. 2016). 


\section{Rearrangement of MA layer occurs during maturation}

In immature particles, the Gag-MA layer is consistently arrayed underneath the inner membrane (Figure 1A, D and 2C), whereas in mature particles where the Gag polyprotein has been

119 proteolytically cleaved, the MA layer appears fragmented (Figure 2G), indicating that MA 120 rearranges during particle maturation. Notably, Env trimers in the mature particles are not

121 colocalized with the MA layer (Figure 2G). Given this ultrastructural reorganization following 122 maturation, it seems possible that disruption of membrane-associated Gag lattices may be a 123 prerequisite for Env trimers to gain necessary mobility to mediate membrane fusion (Wyma, Jiang

124 et al. 2004, Roy, Chan et al. 2013). These structural changes may also provide sufficient membrane 125 pliability for remodeling during viral fusion, similar to matrix layer disruptions seen in other 126 viruses such as influenza virus (Gui, Ebner et al. 2016).

\section{Sub-nanometer HIV-1 Env structure shows a conformationally variable gp41 subunit}

A total of 32802 sub-volumes were used to reconstruct a C3-symmetric sub-tomogram averaged structure of hVLP-Env with local resolution ranging between 5.7 -12 $\AA$ and a global

131 resolution of $9.1 \AA$ (Figure 1C, 3A, B and Figure S3) (Table 1). Classification of the sub-volumes

132 did not yield any other distinct conformational class, indicating that the hVLP-Env were in a 133 predominantly closed, pre-fusion state. The Env ectodomain connects to the membrane via a thin 134 tripod stalk (Figure 1C and 3B). Though the TMD can be observed spanning the membrane layer 135 in raw tomograms (Figure 1A), no evidence of TMD is seen in the sub-tomogram averaged 136 structure (Figure 3B), similar to the Env structure from immature hVLPs. 
et al. 2012, Guttman, Garcia et al. 2014), were well-protected in hVLP-Env (Figure 3C),

140 confirming its closed, pre-fusion conformation. Relative to standard HDX-MS experimental

141 approaches, we were able to improve peptide coverage by performing deuterium exchanges under

142 native conditions but solubilizing the surface protein using detergent under quench conditions

143 (Figure S5). Comparison of hVLP-Env with BG505.SOSIP showed that cognate peptides

144 throughout the trimers had similar exchange profiles (Figure S6), indicating that they both have 145 comparable global conformations. However, the hVLP-Env trimer on virions exhibited greater 146 exchange protection, indicating it is in general more structurally ordered than the engineered 147 soluble trimer (Figure 3C and S6). Indeed, in terms of gp120 subunit organization, hVLP-Env has 148 a global architecture that closely resembles previously published Env structures (Kwon, Pancera

149 et al. 2015, Ward and Wilson 2015, Lee, Ozorowski et al. 2016, Pan, Peng et al. 2020). This is 150 confirmed by good agreement seen in fitting the atomic structure of detergent extracted full-length 151 Env (PDB ID: 6ULC) into the sub-tomogram averaged map (Figure 3A, B).

\section{Differences in gp41's terminal HR2 helix and stalk influence MPER surface exposure} subunit of hVLP-Env. In nearly all known Env structures, the HR2 helix has a long, rod-like

156 conformation with Asp664 forming its distal tip (Kwon, Pancera et al. 2015, Ward and Wilson 157 2015). In contrast, in hVLP-Env, Gln-653 forms the distal tip of the HR2 helix, making the helix 158 length 10 amino acids shorter (Figure 3B). The remaining residues in the C-terminal portion of 159 HR2 helix bend and form a thin stalk connecting the ectodomain to the membrane (Figure 3B). 160 Apart from hVLP-Env, this bend in HR2 helix has only been observed in the full-length, detergent161 solubilized Env structure derived from strain 92UG037.8 (Pan, Peng et al. 2020). In the presence 
162 of membrane, we observe that the thin stalks, corresponding to Env residues 654-664, form a tripod

163 that elevates the ectodomain $\sim 10 \AA$ above the membrane (Figure 3B). As a result, the bulk of the

164 MPER (Membrane-Proximal External Region) (residues 660-683), which is a desirable vaccine

165 target of some of the most broadly neutralizing HIV-1 antibodies isolated to date (Gray, Madiga

166 et al. 2009, Rantalainen, Berndsen et al. 2020), is primarily embedded within the membrane in our

167 structure.

The complete MPER peptide is unresolved in all reported structures of trimeric Env, owing

169 to its membrane proximal location and/or possible variations in its conformation. Current

170 knowledge of MPER structure is only derived from constructs without the Env ectodomain

171 included (Fu, Shaik et al. 2018, Piai, Fu et al. 2020). In a recently published structure of Env from

172 BaL-1 virions, MPER was indicated to form part of the stalk connecting Env ectodomain to the

173 membrane ( $\mathrm{Li}$, Li et al. 2020), which contrasts with the membrane embedded MPER location

174 identified in our structure (Figure S7). However, the embedded nature of the MPER in our map is

175 in agreement with that inferred from other solubilized full-length Env structures and biophysical

176 studies on binding of MPER-targeted antibodies to HIV-1 Env (Kim, Sun et al. 2011, Lee,

177 Ozorowski et al. 2016, Wang, Kaur et al. 2019, Rantalainen, Berndsen et al. 2020). Analyzing Env

178 structures from different HIV strains shows that the general position of the bulk of the ectodomain

179 is $\sim 10-12 \AA$ above the interpreted membrane surface in all cases (Figure S7). However, differences

180 arise in the position of HR2 helix and subsequent MPER sequence leading to variability in MPER

181 presentation and accessibility above the membrane amongst HIV-1 strains. 


\section{A flexible gp41 stalk allows Env orientational freedom and MPER epitope exposure}

In our tomographic data, we observe tilting of hVLP-Env with respect to the membrane

187 (Figure 4A), which leads to a lower density level for the stalk in our sub-tomogram averaged map

188 (Figure 4B). Thus, the tripod stalk of Env is flexible in its native state, allowing differential sampling of MPER and other membrane-proximal epitopes. Although tilting or lifting of Env from

190 the membrane on binding to MPER targeted antibody 10E8 (Lee, Ozorowski et al. 2016, 191 Rantalainen, Berndsen et al. 2020) and gp120-gp41 interface targeting antibody 35022 (Huang,

192 Kang et al. 2014) has been suggested, our results provide the first direct evidence of Env tilting on 193 native membrane in its natural, unliganded state.

Sensitivity to MPER broadly neutralizing antibodies (bnAbs) has been shown to be

195 impacted by Env stability in its prefusion state (Kim, Leaman et al. 2014), with increased neutralization observed after cellular receptor engagement (Frey, Peng et al. 2008, Chakrabarti,

197 Walker et al. 2011). Based on the structural data discussed above, we envision that local structure 198 of HR2, height of the Env ectodomain above membrane, and propensity of Env tilting have 199 additional impact on Env sensitivity or resistance to MPER bnAbs. To test this hypothesis, we 200 conducted comparative neutralization assays using MPER bnAbs against HIV-1 strains whose Env 201 structures have been determined. As anticipated from the structural data, amongst the three viruses, 202 ADA.CM hVLP, BaL-1 and BG505, ADA.CM was overall more resistant to MPER bnAbs (Figure 203 4C and Table S2). Neutralization and mutational analysis of proximal residues in ADA.CM that 204 contribute to trimer stability (Figure S8) showed modest individual effects but none were 205 individually responsible for ADA.CM's enhanced resistance to MPER bnAbs (Figure S8 and 206 Table S3). 
Another prominent difference in the gp41 subunit of hVLP-Env is absence of complete

211 density for the central C-terminal HR1 helices (HR1-C) (Figure 5A). The HR1-C helices

212 comprised of amino acid residues 570-595 have been a hallmark of high resolution Env SOSIP

213 structures with the central trimeric bundle composed of a HR1-C helix from each of the gp41

214 domains. However, in our structure, only partial density for the HR1-C helix can be observed

215 corresponding to gp41 residues 570-577 and 582-595 (Figure 5A). Classification of Env sub-

216 volumes with a tight cylindrical mask for the gp41 region did not yield any subset with higher

217 density for HR1-C helices in our data. Considering that our sub-tomogram averaged map has a

218 local resolution range for the ectodomain between 5.7-12 $\AA$ (Figure S3), it is clear from

219 comparisons with other cryo-EM maps at similar resolutions, that the absence of complete HR1-

220 C helix density in our structure is not an effect of map resolution (Figure S9).

221

To ascertain whether the observed HR1-C density was affected by applied symmetry to the

222 map, we calculated an asymmetric structure of hVLP-Env at $10.7 \AA$ resolution (Figure S3 and

223 Table 1). The asymmetric structure (Figure 5B) appears overall similar to the three-fold

224 symmetrized map (Figure 5A). Partial density for the HR1-C helices is consistently present across

225 all three protomers in the asymmetric structure, with near-complete density for HR1-C observed

226 in one protomer (Figure 5B).

HDX-MS analysis provides additional insight into the state of this key structural motif.

228 Peptides in HR1-C helix (residues 570-579 and 583-593) are more protected in hVLPs than in

229 BG505.SOSIP (Figure 5C), indicating increased backbone hydrogen bonding interactions in 230 corresponding residues. Taken together, the sub-tomogram averaged structures and HDX-MS 
231 analysis shows that the peptides in HR1-C region exist in a stable, ordered conformation in hVLP-

232 Env even though they do not rigidly conform to Env's global three-fold structural symmetry.

234 An extensive and heterogenous glycan shield in hVLP-Env protects critical epitopes

236 decorate the ectodomain surface. In contrast to most available structures where density near to

237 glycosylation sites often covers only the core GlcNAc sugars, in our unliganded hVLP-Env

238 structure, extended electron density for glycan moieties is observed across the protein. Resolved

239 density for multiple canonical glycan positions in gp120 and gp41 subunits extends beyond the

240 range of current structural models, revealing the extent of the 'glycan shield' (Figure 6A) (Wei,

241 Decker et al. 2003). Glycans surrounding a number of key bnAb epitopes can be identified in the

242 unliganded Env map, which reveal their orientations in the natural, antibody-naïve state so as to

243 occlude underlying epitopes.

244 At the CD4 receptor binding site (CD4bs) in hVLP-Env, overlaying the previously

245 determined Fab (Fragment antigen binding) structure of VRC01 (CD4bs bnAb) (Stewart-Jones,

246 Soto et al. 2016) in its binding orientation, highlights possible clashes with nearby glycans N197,

247 N276, N361 and N461 (Figure 6B). These glycans have also been shown to overlap with the

248 VRC01 epitope using molecular dynamic studies, but previously determined structures using

249 SOSIPs resolved only short glycan chains at these sites (except for N276) resulting in minimal

250 apparent glycan interactions with the bnAb (Stewart-Jones, Soto et al. 2016). In hVLP-Env,

251 extended density for these multiple glycans can be seen to clearly deter binding of CD4 epitope-

252 directed nAbs (Figure 6B). These observations are corroborated in VRC01 based neutralization

253 assays which show that hVLP-Env is substantially more resistant than BaL-1 Env (Figure 6B and 
254 Table S2), whose structure lacks extended glycosylation around this site (Li, Li et al. 2020).

255 Indeed, removal of N276 and N461 glycans has been demonstrated to facilitate engagement of

256 germline precursor antibodies for VRC01-like nAbs that normally do not interact strongly with

257 natively glycosylated Env (McGuire, Hoot et al. 2013).

258 Similarly, long-range extended densities for N-linked glycans are observed in the gp41

259 domain corresponding to residues N262, N448, N611 and N637. The close spacing of N262/N448

260 to N611 likely orders them such that they are visible in the averaged map (Figure 6A, C).

261 Collectively, these glycans form a protective barrier over the fusion peptide (FP) in hVLP-Env

262 (Figure 6C). Env's FP is essential for viral cell entry but is surprisingly exposed to solution and

263 highly dynamic by HDX-MS analysis (Figure 5C). Mapping the Fab structure of FP targeting

264 bnAb, PGT151 (Lee, Ozorowski et al. 2016), onto its corresponding epitope on hVLP-Env shows

265 how the glycans would need to be displaced in order for the antibody to bind (Figure 6C). Though

266 hVLPs are neutralized by PGT151, they are relatively resistant compared to other strains (Figure

267 6C and Table S2), suggesting that glycan occlusion may impact neutralization by FP targeted

268 bnAbs as has been reported (Stewart-Jones, Soto et al. 2016).

269 Extensive glycan densities also persist in the asymmetric structure of hVLP-Env but

270 strikingly, we observe variations in densities between different protomers, indicating heterogeneity

271 in glycan occupancy. For example, the N355 glycan is present at all three protomers in the

272 asymmetric Env reconstruction but its density varies with respect to size and orientation at this site

273 (Figure 6D). Similarly, the conserved N88 glycan, which forms part of the epitope of bnAb $35 \mathrm{O} 22$

274 (Huang, Kang et al. 2014) and borders that of 10E8 (Lee, Ozorowski et al. 2016), can only be

275 observed in one protomer but not in others (Figure 6D) and is absent in the C3-symmetrized map.

276 In mass spectrometry analysis of hVLP-Env, these glycan sites, along with others, exhibit presence 
277 of a heterogenous population of complex sugars (Table S4). Thus, though identified heterogeneity

278 in glycan processing of hVLP-Env (Table S4) is similar to previous reports (Guttman, Garcia et

279 al. 2014, Cao, Pauthner et al. 2018, Berndsen, Chakraborty et al. 2020); our comparative structural

280 analysis on asymmetric and symmetric hVLP-Env directly resolves glycan heterogeneity within

281 protomers of the same Env trimer.

282 When comparing bnAbs affected by both the presence of glycan and membrane proximity,

283 binding of bnAb $35 \mathrm{O} 22$ (Huang, Kang et al. 2014) is highly dependent on the presence of N88

284 glycan whereas 3BC176 binding (Lee, Leaman et al. 2015) is partially inhibited by it. Mapping

285 binding orientation of both these Fabs onto hVLP-Env results in steric clashing of the Fab regions

286 with the membrane surface (Figure 6E). Nevertheless, hVLPs are effectively neutralized by $35 \mathrm{O} 22$

287 but show relative resistance to 3BC176 (Figure 6E and Table S2), presumably due at least in part

288 to 3BC176's lower binding capacity (Lee, Leaman et al. 2015). Thus, a combination of

289 heterogenous presence of N88 glycan along with the ability of hVLP-Env to tilt on membrane can

290 account for differential access to these binding epitopes.

291

\section{Conclusions}

In this study, we have analyzed the structure of HIV particles displaying functional Env.

294 Although the ADA.CM Env trimers used are highly stable in a membrane environment, they

295 notably do not form well-ordered gp140 trimers in solution using the typically employed "SOSIP"

296 mutations (Leaman and Zwick 2013). This is true for the majority of Env sequences that require

297 extensive modifications in order to encourage formation of stable ectodomain trimers (Rawi,

298 Rutten et al. 2020). Thus, the structural details discussed here may reflect features of Env strains 
that are not compatible with SOSIP modifications and thus have not been structurally characterized

300 so far.

In hVLP-Env, we find substantial evidence that key parts of gp41 including the HR1 central

302 helices and flexible stalk are not rigidly fixed relative to the rest of the trimer. Indeed, R.M.S.D.

303 calculations among static high-resolution structures of full-length Env and SOSIP structures show

304 higher deviation in the gp41 domain compared to gp120 domain (Table S5), despite the strains

305 having greater than 75\% sequence similarity (Table S6). Likewise, DEER spectroscopy studies of

306 SOSIP trimers have also shown a higher degree of variability in the gp41 domain (Stadtmueller,

307 Bridges et al. 2018), further substantiating the flexible nature of gp41 as inferred from our

308 structures.

309 Our data also demonstrate that even in membrane associated Env, the fusion peptide (FP)

310 is highly dynamic and exposed to solvent. Exposure of such a functionally critical and conserved

311 component seems counter-intuitive, but the dense clustering of glycans around the FP proximal

312 region likely confers a degree of steric protection. However, breaches in the glycan shield, revealed

313 by heterogeneity in glycan density in this region in our structures, can still offer access to the FP

314 by bnAbs (Kong, Xu et al. 2016, Lee, Ozorowski et al. 2016). Moreover, in cases where glycans

315 form part of bnAb epitopes, heterogeneity in glycosylation provide mechanisms exploited by HIV-

3161 as a means of increasing epitope variability and facilitating evasion of antibody responses.

317 Finally, the extent of ordered density between adjacent glycans in hVLP-Env suggests that the

318 glycans are interacting in a stable manner, consistent with recent reports (Stewart-Jones, Soto et

319 al. 2016, Berndsen, Chakraborty et al. 2020).

320 By imaging Env in its native membrane-bound context, we found that, like other type I

321 fusion proteins such as SARS-CoV-2 S trimers (Ke, Oton et al. 2020), HIV-1 Env ectodomain sits 
322 atop a flexible stalk that affords it considerable freedom of motion to tilt relative to the membrane.

323 This likely plays an important mechanistic role by affording fusion proteins the flexibility to bind

324 receptors and refold during membrane fusion. The flexible stalk also impacts the accessibility of

325 key epitopes that are sterically hindered on the membrane-facing side of the trimer ectodomain

326 such as the FP, gp120/gp41 interface, and membrane-associated MPER. Thus, our structural

327 analyses revealed previously uncharacterized Env features that impact presentation of prime

328 epitopes which can help explain differences in neutralization sensitivity across diverse HIV-1

329 strains.

330 Lastly, our structural analyses of Env in immature viral particles, shows that Env is situated

331 directly over two-fold symmetric contacts of the Gag lattice, providing the first structural evidence

332 of a direct physical Env-Gag interaction, helping to inform models for virion particle assembly

333 and Env incorporation. Taken together, our results advance understanding of native HIV-1 Env in

334 the context of virion assembly, maturation and conformational sampling, revealing insights that

335 are generally unavailable through structural studies on recombinant proteins alone.

338 Acknowledgements:

339 We thank the University of Washington Arnold and Mabel Beckman cryoEM center and the

340 School of Pharmacy mass spectrometry center for data collection time and support. This work

341 was supported by National Institutes of Health (NIAID) grants R01GM099989 and

342 R01AI140868 to K.K.L., T32 GM008268 to M.A.B., and R01AI143563 to M.B.Z., the James B.

343 Pendleton Charitable Trust (M.B.Z.), and by the University of Washington's Proteomics

344 Resource (UWPR95794). 
345 Author Contributions: V.M.P.: Conceptualization, Formal analysis, Investigation, Validation

346 and Writing.:D.P.L.: Investigation, Formal Analysis. K.N.L.: Investigation, Formal Analysis.

347 M.A.B.: Investigation. E.A.H.: Investigation. M.B.Z.: Resources, Writing review, Funding

348 acquisition. K.K.L.: Conceptualization, Writing, Funding acquisition.

349

350 Competing interests: Authors declare no competing interests;

351

352 Data Availability: Density maps with corresponding atomic models have been deposited with

353 accession codes EMD-XXXX, EMD-XXX, EMD-XXX, EMD-XXXX and PDB IDs XXXX and

354 XXX.

355 


\section{Figures:}
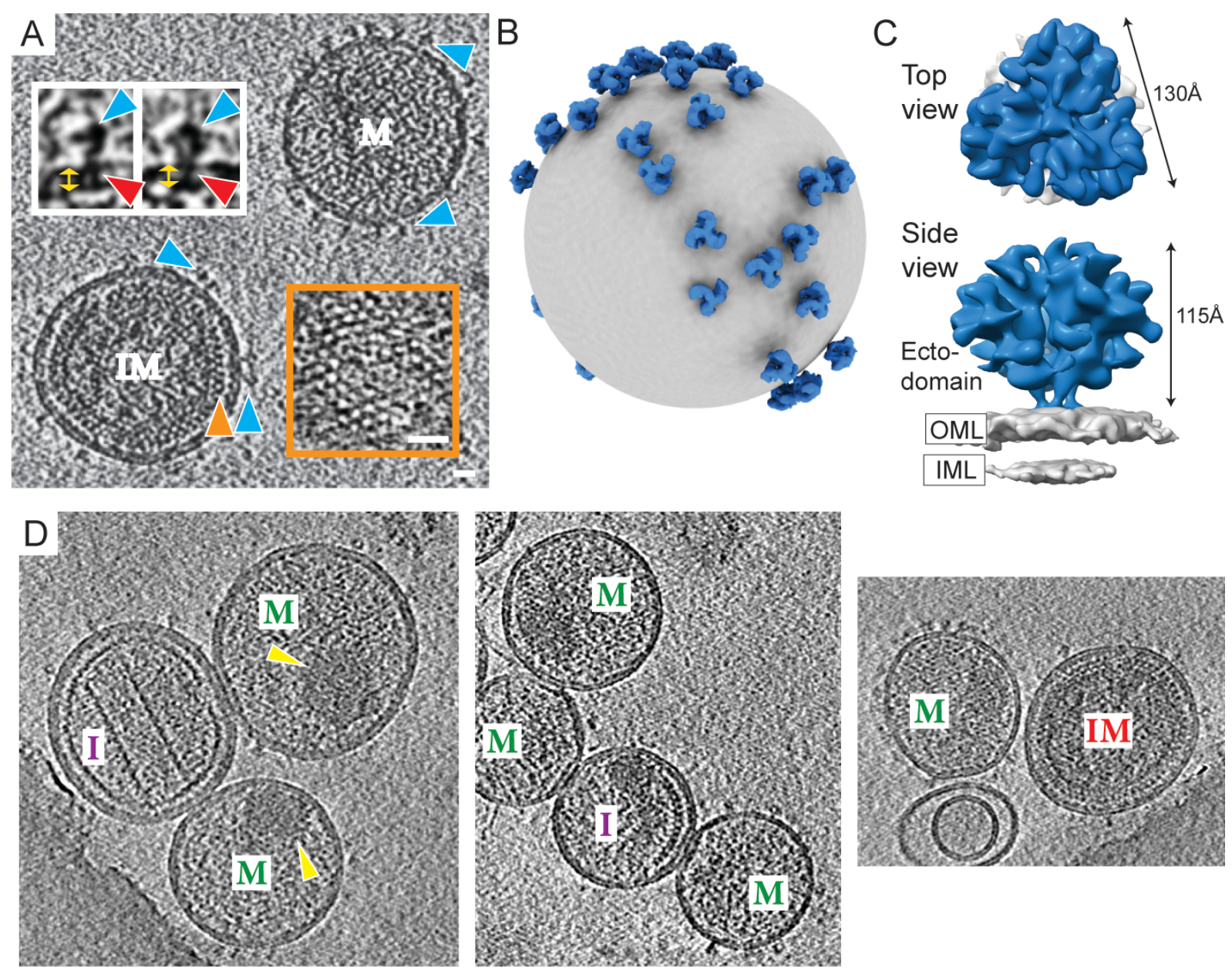

Figure 1. Structural analysis of hVLP-Env. (A) Tomogram slice showing a mature (M) and

359 immature hVLP (IM). Blue arrows indicate Env. Orange arrow indicates Gag layer in IM with zoomed inset of Gag top view on bottom right. Scale bars indicate 200 Å. Top left inset shows

361 close-up of Env with red arrows denoting TMD and yellow arrows denoting membrane bilayer.

362 (For antigenic profile of hVLPs, see Figures S1 and S2). (B) Representative model of mature

363 hVLP with averaged Env structure (in blue) placed onto original particle coordinates. Membrane

364 surface is shown in grey. (C) Sub-tomogram averaged Env structure at 9.1 resolution (for

365 resolution related statistics, see Figure S3). OML and IML indicate outer and inner membrane

366 leaflets, respectively. (D) Tomogram slices showing different maturation states observed in the 
367 hVLP population. Immature particle showing a continuous Gag layer underneath the membrane

368 is labeled IM. Mature particles with the Gag layer reorganized into clearly formed internal capsid

369 is labeled M. Yellow arrows indicate the internal mature capsid in two representative virions.

370 Particles at intermediate stages of maturation are labeled I. Scale bar corresponds to $50 \mathrm{~nm}$.

371 Black is high density in all panels.

372 

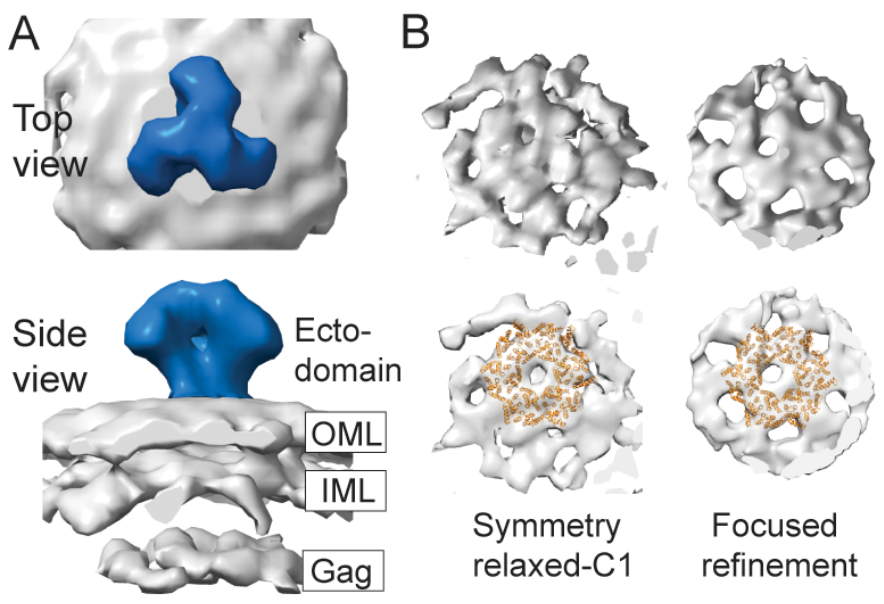

Symmetry relaxed-C1

\section{Focused} refinement
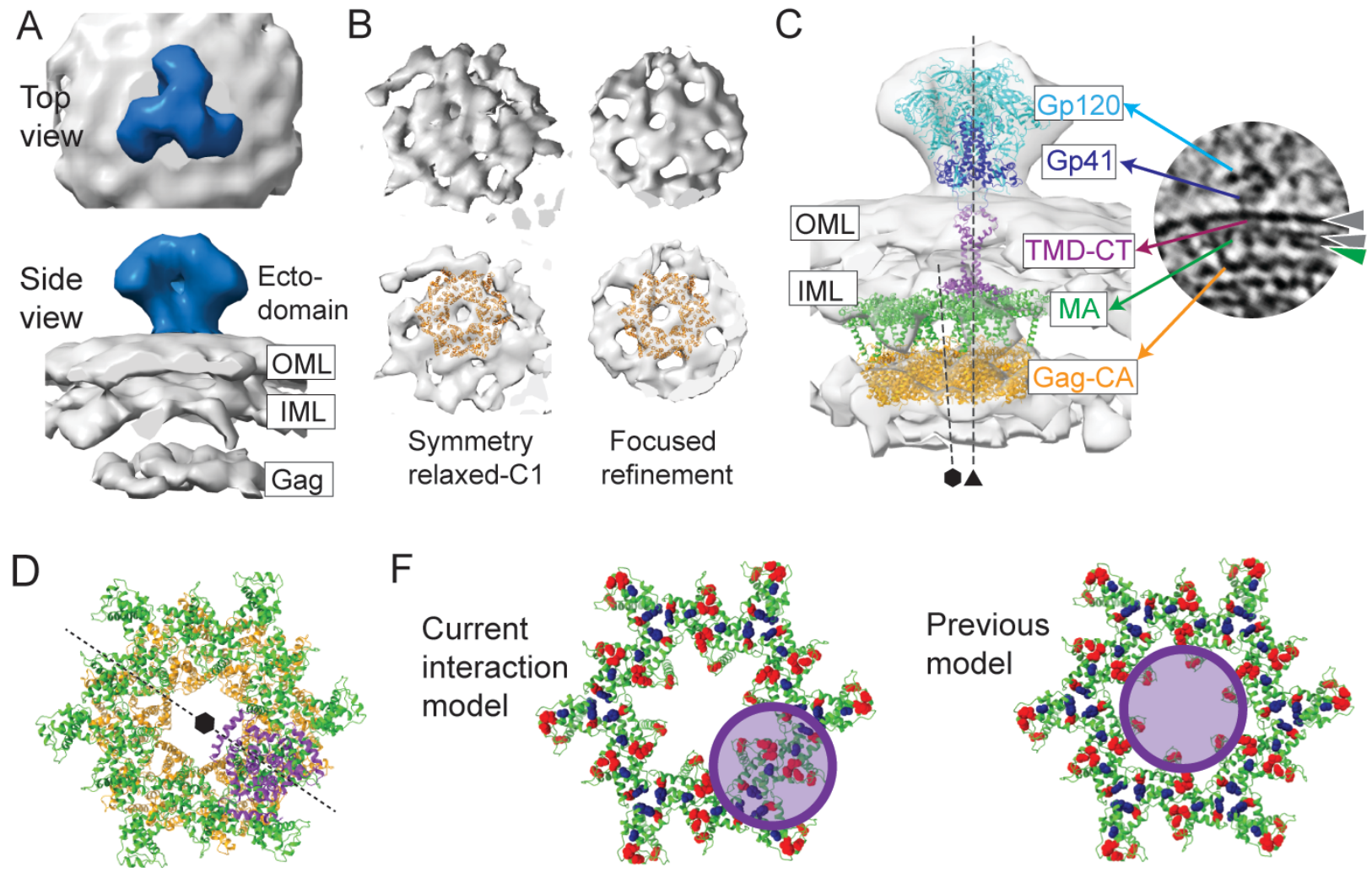

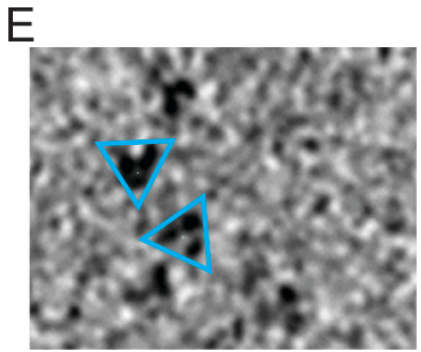

Surface

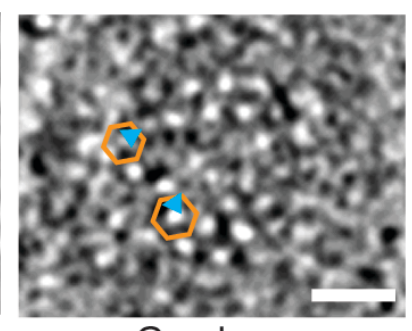

Gag layer

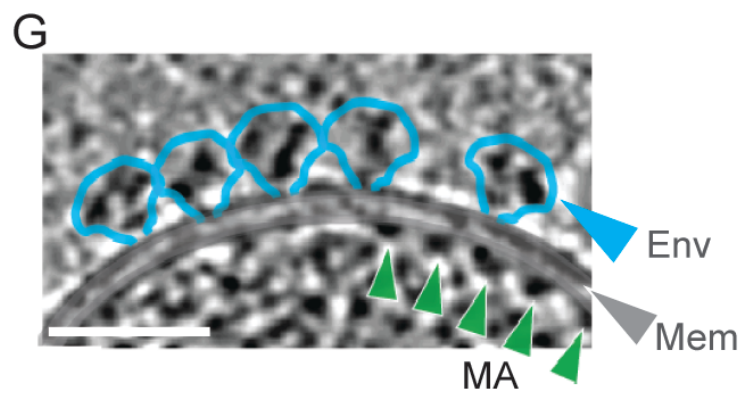

374 Figure 2. HIV Env interaction with immature Gag polyprotein. (A) Sub-tomogram averaged

375 Env structure from immature VLPs. OML and IML indicate outer and inner membrane leaflets,

376 respectively. (B) Top views of immature Gag layer from relaxed C1 symmetry map (top left) and

377 after focused refinement (top right) (see Figure S4 for details). Bottom panels show the same

378 with fitted atomic structure of hexameric Gag-CA (PDB: 4USN). (C) Left: Composite model of

379 Env-Gag structures in immature particles. Atomic coordinates of Env ectodomain with Gp120

380 (cyan) and Gp41 (dark blue), TMD and CTD in purple (PDB: 6UJV), Gag-MA hexamer (PDB:

381 1HIW) in green and Gag-CA hexamer in orange are shown. Right: Tomogram slice of Env from 
an immature particle with protein components labeled. Grey and green arrows indicate membrane bilayer and Gag-MA respectively. (D) Top view of CTD (purple) and Gag-MA-CA

384 (green) interface. CTD lies on the 2-fold axis (black line) of underlying Gag-CA hexamer

385 (orange). (E) Left: Tomogram cross-section of immature VLP surface showing Env positions

386 (blue triangles). Right: Position of Env central axis (blue triangles) with respect to the Gag-CA

387 hexamer (orange hexagon) is shown when viewing along an axis perpendicular to the plane of

388 image. Env position superposes on the edge of Gag hexamer. Black represents high density.

389 Scale bar is $200 \AA$ in length. (F) Model of Env-CTD interaction with Gag-MA. Ribbon diagram

390 of Gag-MA hexamer (green) (PDB ID: 1HIW) (Hill, Worthylake et al. 1996) is shown. Residues

391 that reduce Env incorporation when mutated (13L, 17E, 31L, 35V, 99E and 75LG) are

392 highlighted as red spheres (Alfadhli, Staubus et al. 2019). Residues that reportedly suppress Env

393 incorporation defects (62QR, 35VI and 44FL) are shown as blue spheres (Alfadhli, Staubus et al.

394 2019). Position and area covered by Env CTD structure (based on PDB ID: 6UJV (Piai, Fu et al.

395 2020)) in our current model (left) and previously predicted models (right) is shown as purple

396 circle. In previous models (right panel), Env CTD position was predicted to be at the center of

397 the Gag-MA hexamer. In this configuration, Env CTD directly interacts with only a few residues

398 that line the inner circumference of Gag-MA hexamer. Thus, the effect of the majority of

399 involved residues on Env incorporation would be allosteric. In the current model (left panel)

400 based on our cryo-ET data, the Env CTD position encompasses all residues determined to affect

401 Env incorporation, giving rise to direct contacts between Env CTD and Gag-MA residues. (G)

402 Cross-section of mature VLP shows the presence of Env (blue) on membrane surface (grey)

403 irrespective of the presence of visible MA density (green) underneath. Scale bars are $200 \AA$. 

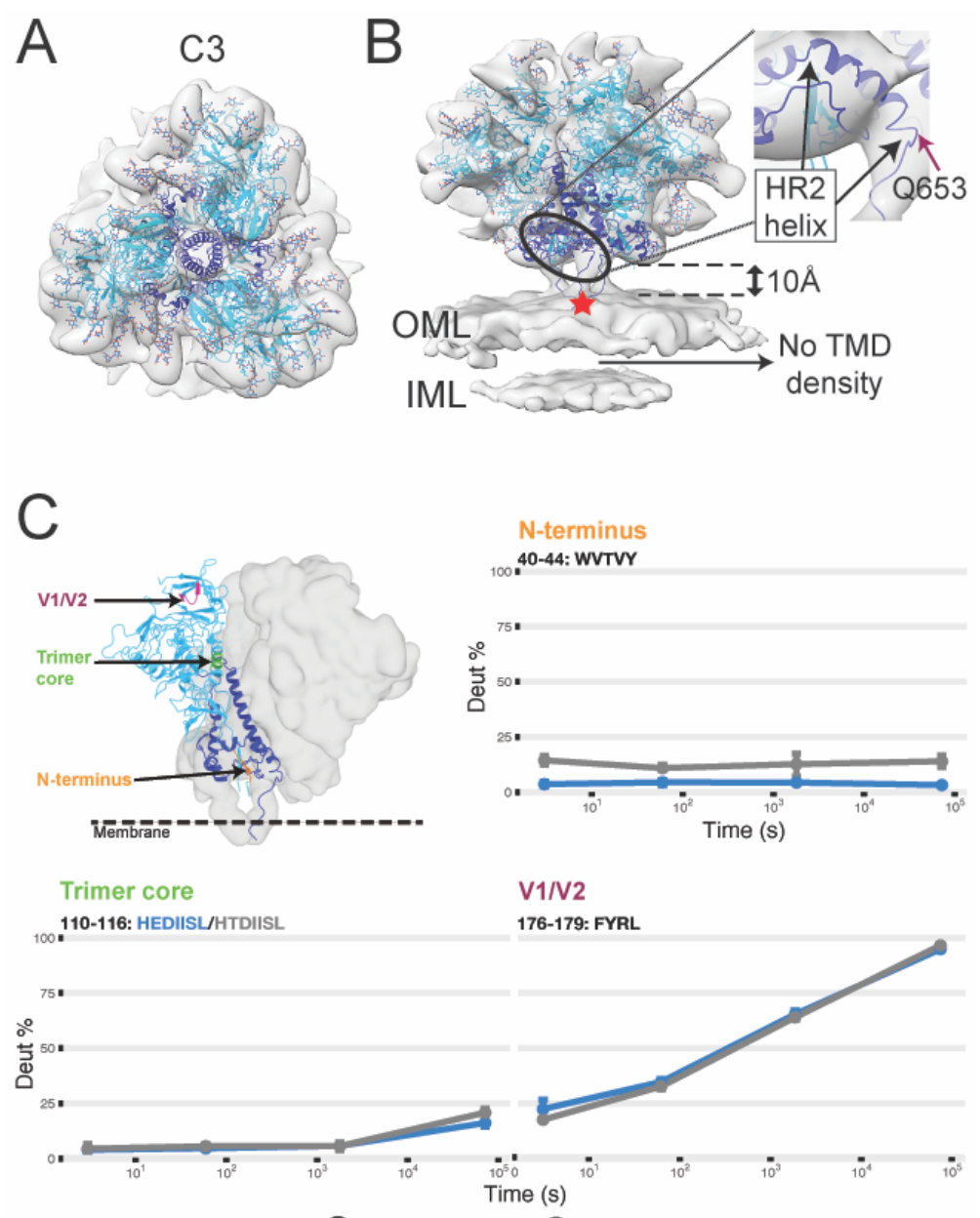

406 Figure 3. Sub-nanometer structure of hVLP-Env indicates conformational variation in HR2

407 helix and MPER location. Top (A) and side-view (B) of sub-tomogram averaged Env map with

408 fitted ribbon structure adapted from full-length Env structure (PDB ID: 6ULC). Gp120 subunit is 409 colored in cyan, gp41 in dark blue, glycans are colored as red and blue heteroatoms. Outer and 410 inner membrane leaflets are indicated as OML and IML respectively. Zoomed inset shows the

411 shortened HR2 helix fitted into its respective density. Red star indicates position of Asp-664 in the

412 trimer structure (See Figure S7 for details on MPER variation). (C) HDX-MS of hVLP-Env shows

413 protection of peptides that report on the closed prefusion conformation of HIV-1 Env (Guttman,

414 Garcia et al. 2014, Guttman, Cupo et al. 2015) (see also Figures S5 and S6). Deuterium uptake for 
415 peptides reporting on trimer integrity behave similarly between hVLP-Env and BG505.SOSIP,

416 which predominantly samples a closed prefusion conformation.

417 


\section{A}
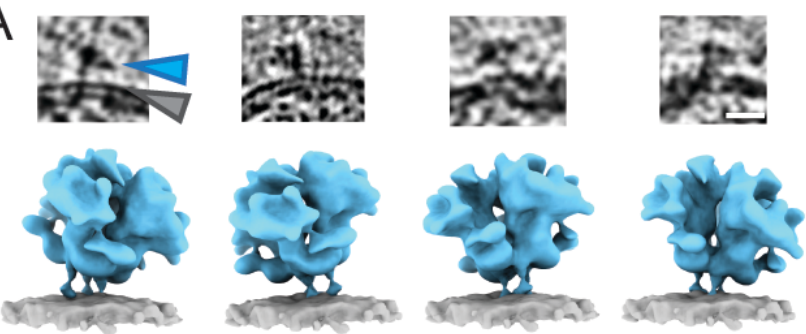

B

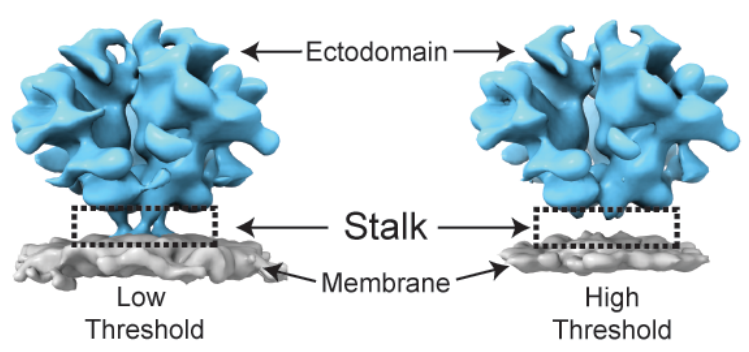

C

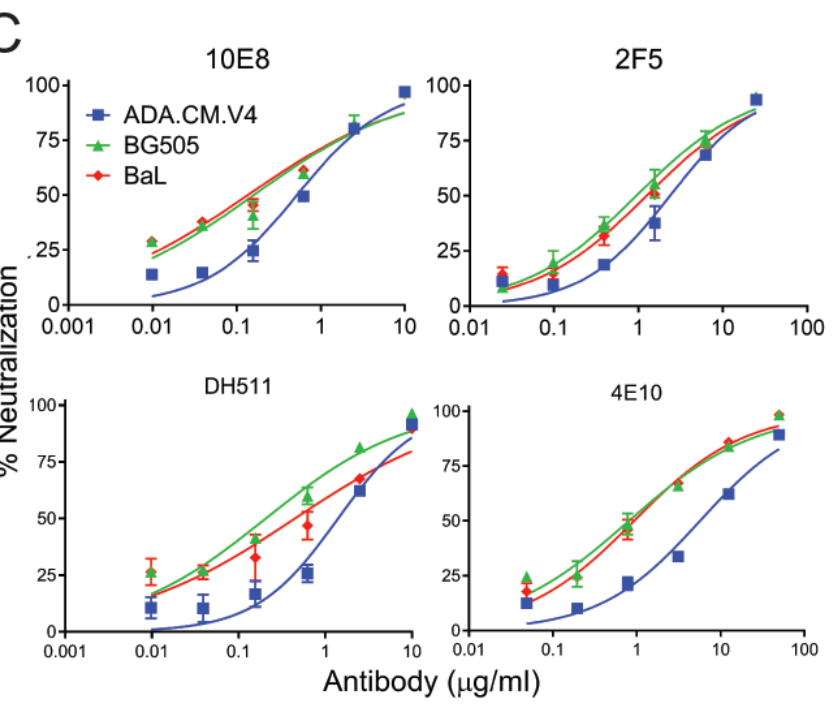

419 Figure 4. Flexible gp41 stalk in HIV Env leads to variation in MPER epitope accessibility.

420 (A) Top: Tomogram slices showing examples of tilted Env on membrane surface. Scale bar equals

421100 Å. Bottom: Surface rendering of modeled Env structure (blue) in tilted orientations on

422 membrane (grey) as seen in the tomograms. (B) Env density map rendered at low and high

423 thresholds, respectively, showing loss of density in the stalk region (black rectangle).

424 Comparison of neutralization effect on different HIV-1 strains by MPER-directed bnAbs. Also see

\section{Figure S8.}




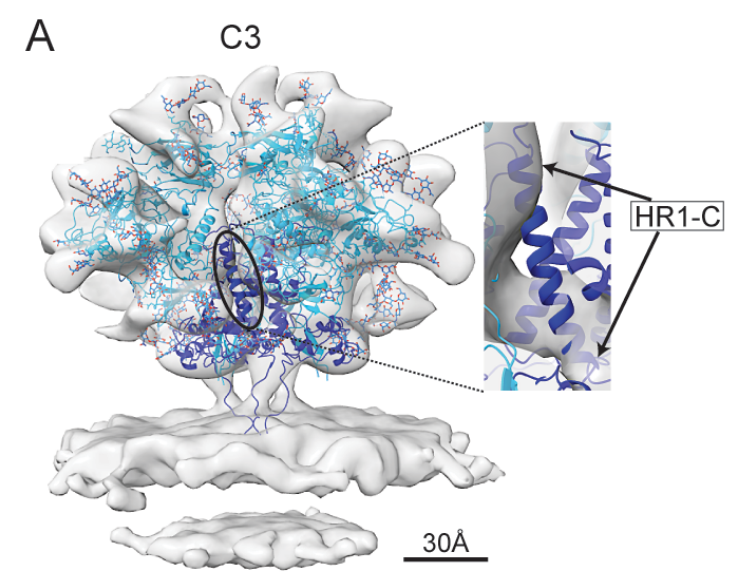

B
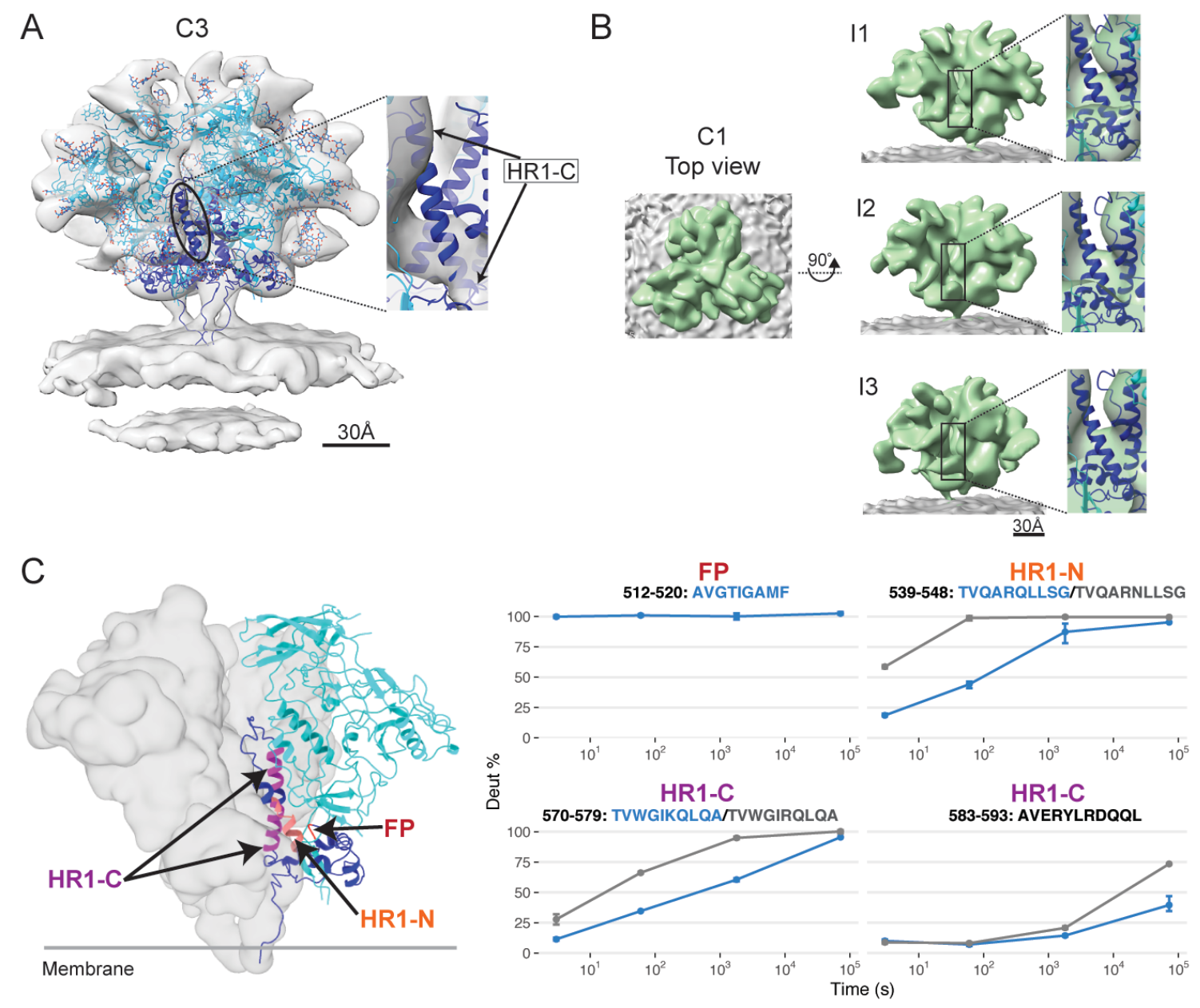

OADA.CM.V4 VLP OBG505.SOSIP.664

428 Figure 5. Conformational variation in central helical bundle (HR1-C) of hVLP-Env. A) Side-

429 view of sub-tomogram averaged Env map with fitted ribbon structure and colored similar to Fig.

430 2. Zoomed inset shows fit of HR1-C helix into its respective density (See also Figure S9). B) Top

431 and side views of an asymmetric reconstruction of hVLP-Env with the three interfaces (I1, I2 and

432 I3) of Env trimer shown along with magnified insets of fitted HR1-C helix. Black, dotted rectangle

433 indicate the central core of the trimer where the HR1-C helix lies. See Figure S3 for resolution-

434 related details. C) HDX-MS deuterium uptake plots for fusion peptide (FP) and HR1 peptides (see

435 also Figures S5 and S6). Surface rendering of Env was calculated using the fitted atomic model of

436 hVLP-Env in panel A without glycans. Full FP region is not observed in hVLP-Env structure due

437 to flexibility. 


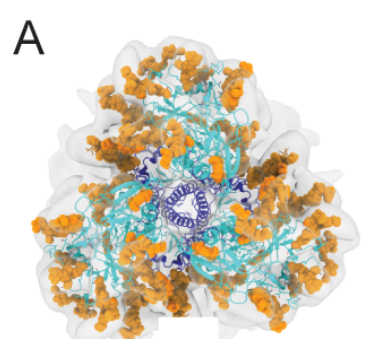

$90^{\circ}+\cdots$

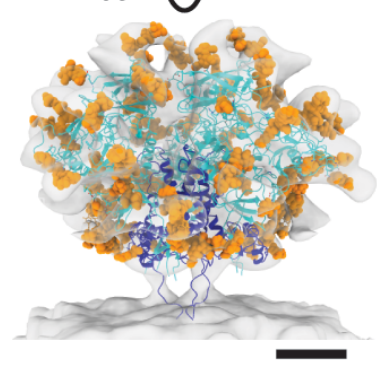

D

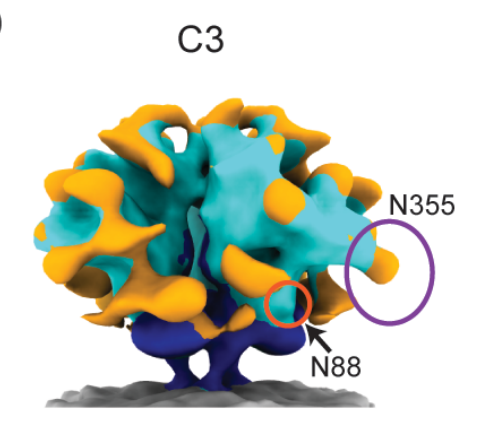

C

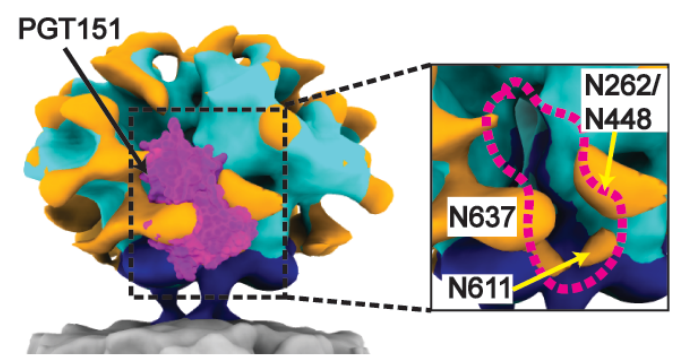

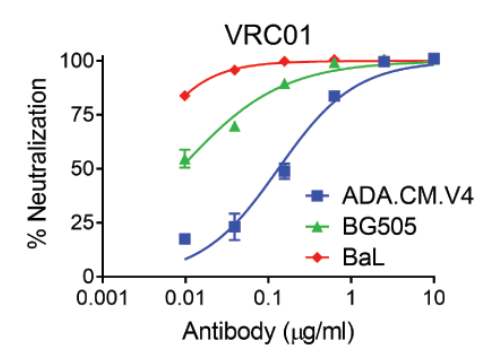

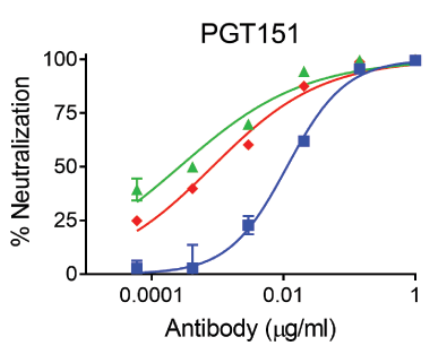

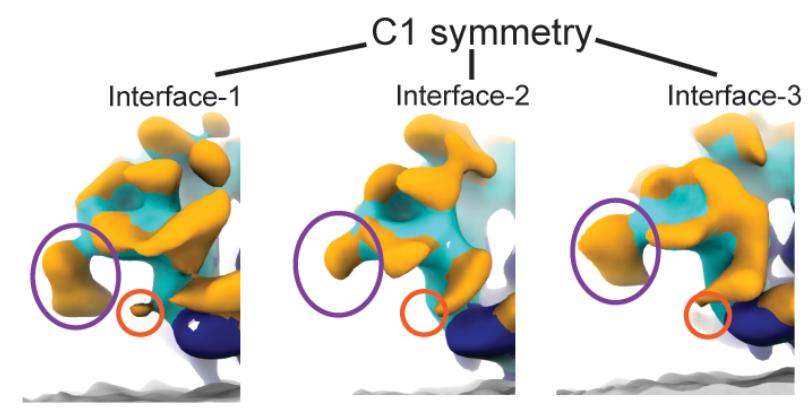

$\mathrm{E}$
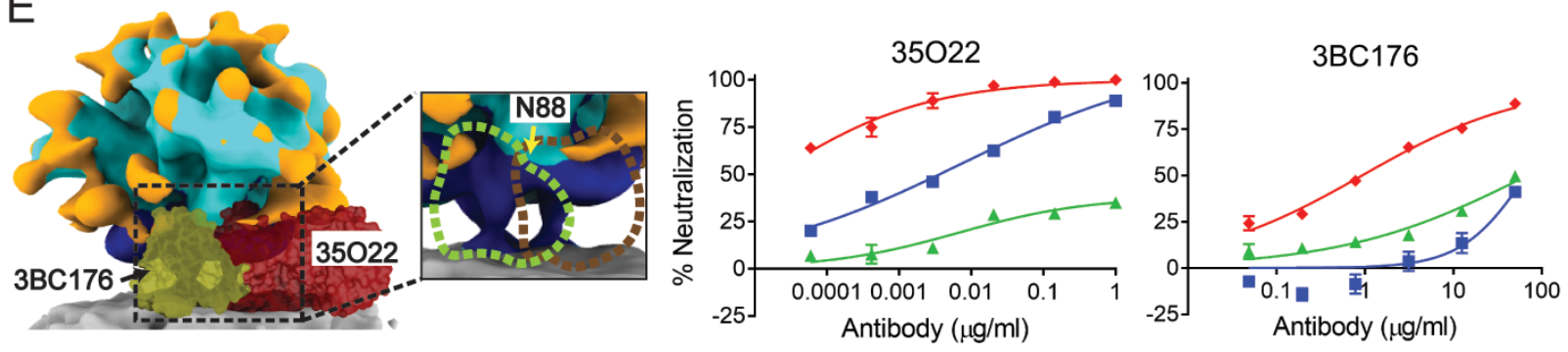

439 Figure 6. Presence of an extensive glycan shield in hVLP-Env. A) Top and side view of

440 hVLP-Env with glycans rendered as orange ball-stick structures. Gp41 colored in dark blue and

441 gp120 in cyan. B), C) and E) Binding positions of four bnAbs - VRC01 (dark grey), PGT151

442 (pink), $35 \mathrm{O} 22$ (brown) and 3BC176 (green) mapped onto hVLP-Env surface. Gp120, gp41,

443 glycan surfaces and membrane are colored in cyan, dark blue, orange and grey respectively.

444 Zoomed-in sub-panel outlines interaction surfaces corresponding to each bnAb. Glycans that 
445 would obstruct bnAb binding are labeled. Neutralization of HIV-1 strains, ADA.CM.V4, BG505

446 and $\mathrm{BaL}$ in presence of each bnAb are shown. D) Differential glycosylation among trimer

447 subunits in hVLP-Env represented using glycans at N88 (orange circle) and N355 (purple oval).

448 Surface rendering of C3-symmetrized map on left followed by the three protomer interfaces from

449 the C1 symmetry hVLP-Env map. Scale bars equal $30 \AA$. 


\section{Table 1.}

451 Data collection and processing parameters for cryo-ET and sub-tomogram averaging.

\begin{tabular}{|c|c|c|c|c|}
\hline \multicolumn{5}{|l|}{ Data Collection Parameters: } \\
\hline Magnification & \multicolumn{4}{|c|}{$58000 X$} \\
\hline Voltage & \multicolumn{4}{|c|}{$300 \mathrm{kV}$} \\
\hline Pixel size & \multicolumn{4}{|c|}{$2.58 \AA ̊$ /pixel } \\
\hline Electron dose & \multicolumn{4}{|c|}{$64-68 \mathrm{e}^{-} / \AA^{2}$} \\
\hline Defocus Range $(\mu \mathrm{m})$ & \multicolumn{4}{|c|}{-2.5 to -5.0} \\
\hline Total number of tilt-series & \multicolumn{4}{|c|}{526} \\
\hline Final number of tilt-series & \multicolumn{4}{|c|}{423} \\
\hline \multicolumn{5}{|l|}{ Data Processing Parameters: } \\
\hline & $\begin{array}{l}\text { hVLP-Env } \\
\text { C3 symmetry } \\
\text { (sym) }\end{array}$ & $\begin{array}{l}\text { hVLP-Env } \\
\text { C1 sym }\end{array}$ & $\begin{array}{l}\text { Immature } \\
\text { hVLP-Env } \\
\text { C3 sym }\end{array}$ & $\begin{array}{l}\text { Immature } \\
\text { hVLP-Env } \\
\text { C1 sym }\end{array}$ \\
\hline Initial number of sub-volumes & 63592 & 63592 & 1520 & 1520 \\
\hline Final number of sub-volumes & 32802 & 29074 & 1051 & 1059 \\
\hline Map resolution at $0.143 \mathrm{FSC}$ & $9.13 \AA$ & $10.67 \AA$ & $31.35 \AA$ & $34.3 \AA$ \\
\hline
\end{tabular}




\section{Experimental Methods:}

454 hVLP expression and purification:

455 Stable cell line expressing high levels of HIV Env, ADA.CM.V4, was described previously (Stano,

456 Leaman et al. 2017). A stable cell line expressing Env BG505 was generated using a similar 457 method, namely by transduction of HEK293T cells using a lentiviral vector 458 (pLentiIII.BG505.755*) containing the env gene BG505 with a stop codon at position 756. 459 Transduced cells were subjected to two rounds of sorting by FACS using fluorescently labeled 460 quaternary neutralizing antibody PGT145 and non-neutralizing CD4BS antibody b6, then gating 461 cells for PGT145 ${ }^{\text {high }}$ b6 ${ }^{\text {low }}$. Env copy number on BG505 VLPs was notably lower than on 462 ADA.CM.V4 hVLPs. Additionally, BG505 VLPs were also more fragile, which precluded 463 extensive cryo-ET data collection and HDX-MS analysis.

470 by layering iodixanol in $1.2 \%$ increments, and centrifuged at $200,000 \times \mathrm{g}$ for $1.5 \mathrm{~h}$ at $4^{\circ} \mathrm{C}$ in an

471 SW41Ti rotor (Beckman). Fractions $(1 \mathrm{ml}$ each) were collected starting from the top and fractions

472 6-9 were saved and pooled. Purified hVLPs were brought up to $15 \mathrm{ml}$ with PBS and concentrated

473 to $\sim 0.2 \mathrm{ml}$ using a $100 \mathrm{kDa}$ MWCO Amicon centrifugal filter (Millipore). VLPs were inactivated

474 by adding aldrithiol-2 (AT-2) to a final concentration of $2.5 \mathrm{mM}$ and samples were incubated at

475 RT for $2 \mathrm{~h}$. The volume was again increased to $15 \mathrm{ml}$ with PBS and hVLPs were concentrated 
476 using a $100 \mathrm{kDa}$ MWCO centrifugal filter to 250-fold the concentration in the original transfection

477 supernatant.

478

479 Cryo-ET sample preparation and tilt-series acquisition:

480 Purified hVLP samples were mixed with 10nm gold beads (Aurion BSA Gold Tracer 10nm) at a 481 ratio of 15:1 (v/v). Using a Vitrobot Mark IV (FEI Co.), $3 \mu$ of this mixture was applied to glow

482 discharged, C-Flat 1.2/1.3, 200mesh or 400mesh grids (Electron Microscopy Sciences), blotted for

483 4-5 seconds and plunge frozen in liquid ethane. Vitrobot was maintained at $4^{\circ} \mathrm{C}$ and $100 \%$

484 humidity during the experiment.

485 Frozen grids were imaged using a $300 \mathrm{kV}$ Titan Krios with a Gatan K2 direct electron 486 detector and GIF energy filter with slit width of $20 \mathrm{eV}$. Tilt-series were collected in a dose487 symmetric tilting scheme from $-54^{\circ}$ to $+54^{\circ}$ or from $-48^{\circ}$ to $+48^{\circ}$ with a step size of $3^{\circ}$ using 488 Leginon (Carragher, Kisseberth et al. 2000) or SerialEM softwares (Mastronarde 2005). Tilt-series 489 were collected in counting mode at a magnification of 53000X, corresponding to a pixel size of $4902.58 \AA$ per pixel. The total dose per tilt series was $\sim 64-68 \mathrm{e}^{-} / \AA^{2}$. A total of 526 tilt-series were 491 collected across multiple sessions.

493 Tomogram reconstruction:

494 Tilt-series image frames were corrected for beam-induced motion using motioncor2 (Zheng, 495 Palovcak et al. 2017). Using batch tomography via the Etomo interface in IMOD software package 496 (Kremer, Mastronarde et al. 1996, Mastronarde and Held 2017), tilt-series were processed for 497 tomogram generation using standard procedures. Tilt series images were aligned using gold bead 498 markers and aligned tilt-series were used to generate a three-dimensional volume using weighted 
499 back-projection method. The final tomograms were rotated, binned, and low pass-filtered for

500 visualization. Tilt-series with non-optimal alignments were discarded. In the end, 423 tilt-series

501 were selected for further processing.

502

503 Initial model generation:

504 Eight tilt-series were randomly selected. CTF estimation and correction for these were done using 505 the Ctfplotter program (Xiong, Morphew et al. 2009). Tomograms were then generated for these 506 CTF-corrected tilt-series in IMOD at the unbinned pixel size (Kremer, Mastronarde et al. 1996, 507 Mastronarde and Held 2017). The aligned tilt-series, reconstructed tomograms, file listing the tilt 508 angles and electron dose values were imported into Relion software suite (Scheres 2012) according 509 to its conventions (Bharat and Scheres 2016). Using the 3dmod graphical user interface (Kremer, 510 Mastronarde et al. 1996), 2067 Env particles on the surface of hVLPs were manually picked. The 511 picked particle coordinates were imported into Relion (Scheres 2012) and corresponding sub-

512 volumes extracted. These were then subjected to 3D classification with a spherical mask covering 513 small parts of the membrane using C1 and C3 symmetry with no initial model provided. Both the $514 \mathrm{C} 1$ and C3 symmetry classifications resolved a 3D class that looked similar to the expected 515 structure of HIV-1 Env. The 3D class from the C3 symmetry run showing clear Env density 516 contained a total of 811 sub-volumes and was selected as initial model.

518 Sub-tomogram averaging:

519 For C3-symmetrized hVLP-Env structure:

520 Tilt-series were imported into EMAN2's sub-tomogram averaging pipeline (Chen, Bell et al. 521 2019). 1k X 1k tomograms were generated within EMAN2 using default parameters. The binned 
522 tomograms were then used for semi-automated particle picking in PEET software (Nicastro,

523 Schwartz et al. 2006). Consolidated particles from the semi-automated picking were further

524 curated manually using the 3 dmod interface (Kremer, Mastronarde et al. 1996) to remove wrongly

525 positioned particle points such as those that were in the membrane or on the inside of VLPs.

526 Curated particle coordinates (63592 particles) were then imported into EMAN2 through the

527 e2spt_boxer.py interface (Chen, Bell et al. 2019). CTF-estimation for all the tilt-series and

528 subsequent CTF-corrections were carried out within EMAN2. Sub-volumes were initially

529 extracted at 4xbinning corresponding to a pixel size of $10.32 \AA$ per pixel. Sub-tomogram

530 refinement was carried using a spherical mask including Env on surface and a part of the

531 membrane. The 3D structure previously generated in Relion (Scheres 2012, Bharat and Scheres

532 2016) was used as initial model after low pass filtering to $60 \AA$. Sub-volumes were then re-

533 extracted at $2 \mathrm{X}$ binning using the particle orientations from the $4 \mathrm{X}$ binned refinement output. Sub-

534 tomogram refinement was repeated using the $2 \mathrm{X}$ binned data with a cylindrical mask covering

535 only the ectodomain and outer membrane layer. Particle orientations were locally refined within a

$53630^{\circ}$ angular limit from the positions calculated in the 4 Xbin refinement. Sub-volumes closer than

$537120 \AA$ when measured between centers were removed. Sub-volumes with low cross-correlation

538 scores were also removed according to EMAN2's default settings. Remaining particles were then

539 re-extracted at the original, un-binned pixel size and re-refined starting from previously determined

540 orientations at $2 \mathrm{X}$ binning. Sub-tilt refinement in EMAN2 was then carried out using default

541 parameters following sub-tomogram refinement with un-binned sub-volumes (Chen, Bell et al.

542 2019). For sub-tilt refinement, a threshold mask was used that enclosed only the Env ectodomain

543 portion without any membrane density. The threshold mask was generated using the mask creation 
544 tool in the Relion package (Scheres 2012). The final masked ectodomain map contained 32802

545 sub-volumes with a calculated resolution of $9.13 \AA$ at 0.143 FSC cut-off value.

546

547 For asymmetric hVLP-Env map:

548 The initial sub-volumes used for generation of the asymmetric map were the same as that

549 used for the C3 symmetrized map described above. Refinement strategies were also nearly

550 identical between the two structures except the asymmetric map was generated with $\mathrm{C} 1$ symmetry.

551 However, the refinement and processing steps were carried out completely independent of each

552 other. The number of sub-volumes in the final C1 hVLP-Env structure was 29074 with a global

553 resolution of $10.67 \AA$ at 0.143 FSC cut-off.

554

555 Structure of hVLP-Env and Gag layer from immature virions:

556 A total of 1520 sub-volumes from only immature virions were used to generate $\mathrm{C} 3$ and $\mathrm{C} 1$

557 symmetrized maps of membrane bound Env using 2Xbinned data in EMAN2 (Chen, Bell et al.

558 2019) by similar procedures as described above.

559 In the unmasked maps, a third density layer was observed underneath the membrane

560 bilayer. Relaxing symmetry of the C3-density map to C1, showed a more defined organization in

561 the Gag layer. Hence, a short, cylindrical mask enclosing only the Gag layer was used for local

562 refinement of the Gag layer using C1 symmetry, starting from the final refined positions derived

563 from the C3-symmetrized immature Env map. This focused refinement gave rise to a $23 \AA$ map of

564 the Gag protein layer (0.143 FSC cut-off). The refined Gag-CA density map was fitted back into

565 the Gag-CA layer of the relaxed to C1 symmetry full Env map for further analyses. 
Sub-tomogram averaged structure of BG505-Env from VLPs: at a ratio of 15:1 (v/v). The mixture was applied to C-Flat grids and plunge frozen using a Vitrobot

570 Mark IV (FEI Co.) similar to the procedure used for hVLPs above.

572 detector and GIF energy filter with slit width of $20 \mathrm{eV}$. Tilt-series were collected in a dose573 symmetric tilting scheme from $-54^{\circ}$ to $+54^{\circ}$ with a step size of $3^{\circ}$ using Leginon (Carragher,

574 Kisseberth et al. 2000) software. Tilt-series were collected in counting mode at a magnification of

$57553000 X$, corresponding to a pixel size of $2.58 \AA$ per pixel. The total dose per tilt series was $\sim 64-$

$57668 \mathrm{e}^{-/} / \AA^{2}$. A total of 49 tilt-series were collected.

577 Tilt-series were imported into EMAN2's sub-tomogram averaging pipeline (Chen, Bell et 578 al. 2019). 1k X 1k tomograms were generated within EMAN2 using default parameters. Particle 579 points were picked manually in the e2spt_boxer.py interface (Chen, Bell et al. 2019). Further 580 refinement and processing steps were carried out similar to the hVLP-Env sub-tomogram 581 averaging procedures described above. Briefly, sub-volumes were initially extracted at 4xbinning 582 corresponding to a pixel size of $10.32 \AA$ per pixel. Sub-tomogram refinement was carried using a 583 spherical mask including Env on surface and a part of the membrane. The initial model generated 584 for hVLP-Env in Relion (Scheres 2012, Bharat and Scheres 2016) was used as initial model for 585 BG505-Env also with low pass filtering to $60 \AA$. Sub-volumes were then re-extracted at $2 \mathrm{X}$ binning 586 using the particle orientations from the $4 \mathrm{X}$ binned refinement output. Sub-tomogram refinement 587 was repeated using the $2 \mathrm{X}$ binned data with a cylindrical mask covering only the ectodomain and 588 outer membrane layer. Particle orientations were locally refined, and duplicates were removed. 589 Sub-tilt refinement in EMAN2 was carried out using default parameters (Chen, Bell et al. 2019). 
590 The final map contained 2773 sub-volumes with a calculated resolution of $16.7 \AA$ at 0.143 FSC

591 cut-off value.

592

593 Model fitting and glycan modeling:

595 (Pan, Peng et al. 2020) was fitted as a rigid body into the $9.13 \AA$ resolution hVLP-Env map. Each

596 protomer, comprising of gp120-gp41 heterodimer, was fitted individually into the map using

597 UCSF Chimera (Pettersen, Goddard et al. 2004). The unstructured loop region at the end of gp41,

598 comprising of amino acid residues 654-664, was fitted into the clearly delineated stalk density

599 using 'Flexible fitting' feature in Coot software (Emsley, Lohkamp et al. 2010). For modelling

600 glycan moieties, previously published high-resolution Env structures, PDB IDs: 5FUU and 5FYJ-

601 L, containing well-built glycan models were used as reference (Lee, Ozorowski et al. 2016,

602 Stewart-Jones, Soto et al. 2016). Appropriate glycan chains from these structures were copied into

603 corresponding glycan positions in the hVLP-Env map based on the presence of unoccupied

604 density. In regions where the unoccupied density was larger than the modeled glycan chains

605 available from the reference PDB structures, these extra densities were left un-modeled.

The final model of hVLP-Env, as generated above, was used for rigid body fitting into the

607 other sub-tomogram averaged maps of asymmetric hVLP-Env and immature hVLP-Env. All rigid

608 body fitting procedures were carried out using UCSF Chimera (Pettersen, Goddard et al. 2004).

609 Root mean square deviation (R.m.s.d) calculations between pairs of HIV Env structures

610 was carried out using UCSF Chimera (Pettersen, Goddard et al. 2004). Multiple sequence

611 alignments were carried out using the Clustal Omega web service (Madeira, Park et al. 2019). 
Structure and tomographic images were generated using UCSF Chimera (Pettersen,

613 Goddard et al. 2004), ChimeraX (Pettersen, Goddard et al. 2021), IMOD’s graphical user interface

614 (Kremer, Mastronarde et al. 1996) and ImageJ (Schneider, Rasband et al. 2012).

615

616 Neutralization assays:

617 ADA.CM.V4 hVLPs were prepared for neutralization assays as described above except omitting 618 the AT-2 inactivation step. Pseudotyped viruses were similarly produced by co-transfection of 619 HEK 293T cells using pSG3 $\triangle$ Env backbone plasmid and Env-complementation plasmid. Serial 620 dilutions of antibodies were added to virus and the mixture was incubated for $1 \mathrm{~h}$ at $37^{\circ} \mathrm{C}$ prior to

621 addition to TZM-bl target cells. DEAE-dextran was added to wells to a final concentration of 10 $622 \mu \mathrm{g} / \mathrm{ml}$. After incubating for $72 \mathrm{~h}$ at $37^{\circ} \mathrm{C}$, cells were lysed, Bright-Glo luciferase reagent (Promega) 623 was added, and luminescence was measured using a Synergy H1 microplate reader (Bio-Tek).

625 Virus ELISA:

626 ELISAs on directly immobilized hVLPs were performed as previously described (Tong, Crooks 627 et al. 2012). Briefly ADA.CM.V4 hVLPs (prepared as detailed above and treated with or without 628 AT-2) were immobilized on microwell plates at a $20 \mathrm{x}$ concentration for $2 \mathrm{~h}$ at $37^{\circ} \mathrm{C}$. Plates were 629 blocked with $4 \%$ non-fat dry milk (NFDM) PBS for $1 \mathrm{~h}$ at $37^{\circ} \mathrm{C}$, probed with serial dilutions of 630 primary antibodies and, subsequently, goat anti-human-Fcy-HRP secondary antibody (Jackson) in 631 PBS $+0.4 \%$ NFDM, and with washes using PBS between each step (detergent was omitted from 632 all steps). Signal was developed using One-step Ultra TMB Substrate (ThermoFisher). 
BG505.SOSIP expression and purification:

635 Soluble BG505.SOSIP protein was expressed and purified as previously described (Verkerke,

636 Williams et al. 2016). Expi293F cells grown in Freestyle 293 media (Thermo Scientific) were co-

637 transfected with plasmids containing the SOSIP sequence and furin protease in a 3:1 ratio using

638 PEI MAX (Polysciences). Six to seven days post-transfection the supernatant was cleared by

639 filtration and the SOSIP purified by Galanthus nivalis lectin affinity, followed by ion-exchange

640 and hydrophobic interaction chromatography. Trimeric product was buffer exchanged into PBS

641 and stored at $4^{\circ} \mathrm{C}$.

642

643 Hydrogen-deuterium mass-spectroscopy (HDX-MS) of ADA.CM.V4 particles and

644 BG505.SOSIPs:

645 HDX-MS using quench lysis method:

646 Frozen stocks of ADA.CM.V4 VLP containing approximately $0.5 \mathrm{mg} / \mathrm{mL}$ envelope protein or

647 purified BG505.SOSIP diluted to the same concentration were buffer-exchanged into HEPES-

648 buffered saline (HBS, 10mM HEPES-KOH, pH 7.4, 150mM NaCl) using Zeba Spin columns with

649 a $7 \mathrm{kDa}$ MWCO (Thermo Fisher). $10 \mu \mathrm{L}$ of VLP solution was diluted with $90 \mu \mathrm{L}$ of deuterated HBS

650 (Hepes Buffer Saline, $\mathrm{pH}$ 7.4) for the indicated labeling time. The reaction was quenched and lysed

651 by addition of an equal volume of $0.2 \%$ formic acid, $4 \mathrm{M}$ guanidine hydrochloride, $0.2 \mathrm{M}$ TCEP

652 (tris(2-carboxyethyl)phosphine), 0.2\% DDM (n-Dodecyl-B-D-maltoside), followed by 30s

653 incubation on ice. $20 \mu \mathrm{L}$ of a $300 \mathrm{mg} / \mathrm{mL}$ solution of $\mathrm{ZrO}_{2}$ beads (HybridSPE-Phospholipid)

654 (Adhikary, Deredge et al. 2017) in $0.1 \%$ formic acid was then added to the solution, followed by

655 vortexing for 30 seconds on ice. The bead/protein mixture was then moved to a $0.22 \mu$ m cellulose

656 acetate centrifuge filter tube (Spin-X, Corning) and spun for 30 s at $13,000 x G, 0^{\circ} \mathrm{C}$. The flow- 
657 through was transferred to a thin-wall PCR tube and frozen in liquid nitrogen. Samples were

658 thawed on ice and passed over a custom packed pepsin column $(2.1 \times 50 \mathrm{~mm}) \mathrm{kept}$ at $15^{\circ} \mathrm{C}$ with a

659 flow of $0.1 \%$ trifluoroacetic acid (TFA), $2 \%$ acetonitrile (ACN) at $200 \mu \mathrm{L} / \mathrm{min}$ for 5 minutes.

660 Digested peptides were collected on a Waters XSelect CSH C18 XP VanGuard Cartridge,

$661130 \AA, 2.5 \mu \mathrm{m}, 2.1 \mathrm{~mm}$ X $5 \mathrm{~mm}$ before separation on a Waters ACQUITY UPLC Peptide CSH

662 C18 Column, $130 \AA, 1.7 \mu \mathrm{m}, 1 \mathrm{~mm}$ X $100 \mathrm{~mm}$ using a gradient of 7 to $14 \%$ B over 1 minute, 14

663 to $30 \%$ B over 12.5 minutes, and 30 to $50 \%$ B over 1 minute, followed by washing with three rapid

664 gradients between 95 and 5\% B (A: 0.1\% formic acid, 0.025\% trifluoroacetic acid, 2\% acetonitrile;

665 B: $0.1 \%$ formic acid in $100 \%$ acetonitrile). The liquid chromatography system was coupled to a

666 Waters Synapt G2-Si Q-TOF with ion mobility enabled. Source and de-solvation temperatures

667 were $70^{\circ} \mathrm{C}$ and $130^{\circ} \mathrm{C}$ respectively. The StepWave ion guide settings were tuned to prevent non-

668 uniform gas phase proton exchange in the source (Guttman, Wales et al. 2016).

669 During the separation step, a series of $250 \mu \mathrm{L}$ injections were used to clean the pepsin

670 column: (1) Fos-choline-12 (Anaspec) in 0.1\% TFA; (2) $2 \mathrm{M} \mathrm{GuHCl}$ in $0.1 \%$ TFA; (3) $10 \%$ acetic

671 acid, 10\% ACN and 5\% IPA (Majumdar, Manikwar et al. 2012, Hamuro and Coales 2018). After

672 each gradient, the trap column was washed with a series of $250 \mu \mathrm{L}$ injections: (1) $10 \% \mathrm{FA}$; (2)

$67330 \%$ trifluoroethanol; (3) 80\% MeOH; (4) 66\% 2-propanol, 34\% ACN; (5) 80\% ACN (Fang, Rand

674 et al. 2011).

675

676 HDX-MS using solution digestion method:

677 Env on hVLPs was quantified by SDS-PAGE using BG505 SOSIPs as a standard. HDX-MS

678 reactions were initiated by diluting $20 \mathrm{uL}$ of either hVLPs or BG505 SOSIPs with $180 \mathrm{uL}$ 679 deuteration buffer (10 mM Phosphate, $150 \mathrm{mM} \mathrm{NaCl}, 85 \% \quad \mathrm{D}_{2} \mathrm{O}$ (Cambridge Isotope 
680 Laboratories)) to a final $\mathrm{pH}=7.45$. Samples were deuterated for 5 seconds, 60 seconds, 30 minutes,

681 or 3 hours before being diluted 1:1 with ice cold quench buffer (8 M urea, $200 \mathrm{mM}$ TCEP [tris(2-

682 carboxyethyl) phosphine] and $0.2 \%$ formic acid (FA)) to a final $\mathrm{pH}$ of 2.5. Quenched samples were

683 digested with $30 \mathrm{ug} / \mathrm{mL}$ of porcine pepsin (Worthington Labs) under quench conditions for 5

684 minutes on ice. Labeled peptides were purified by high speed centrifugation at $0^{\circ} \mathrm{C}(2$ minutes at

$68525,000 \mathrm{rcf}$ ) and immediately flash frozen in liquid nitrogen. BG505 SOSIP samples were handled

686 identically to ensure consistent labeling and back exchange. Frozen samples were stored at $-80^{\circ} \mathrm{C}$

687 until analysis. All reactions were performed in duplicate.

688 Samples were thawed for 5 minutes on ice and manually injected into a custom built HDX

689 LC system kept at $0^{\circ} \mathrm{C}$ using a $500 \mathrm{uL}$ sample loop. Samples were trapped on a Waters ACQUITY

690 UPLC CSH C18 VanGuard $130 \AA, 1.7 \mu \mathrm{m}, 2.1 \mathrm{~mm}$ by $5 \mathrm{~mm}$ trap column for 7 minutes with a flow

691 of solvent A [2\% acetonitrile, $0.1 \%$ FA, $0.025 \%$ trifluoroacetic acid (TFA)] at a rate of 150

$692 \mu \mathrm{L} / \mathrm{min}$. Peptides were resolved over a Waters ACQUITY UPLC CSH C18 130Å, $1.7 \mu \mathrm{m}, 1$ x 100

$693 \mathrm{~mm}$ column using a 20 minute linear gradient of 3\% to 50\% solvent B (Solvent B: 100\%

694 acetonitrile and 0.1\% FA) and analyzed using Waters Synapt G2-Si Q-TOF as described above.

695 Following each injection, the sample loop and trap were washed as described above.

Deuterium uptake analysis was performed with HD-Examiner (Sierra Analytics) and HX-

697 Express v2 (10.1021/jasms.8b04435). For solution digestion experiments data was extracted using

698 CDCReader and analyzed using HXExpress V2 (Guttman, Weis et al. 2013) with binomial fitting 699 and bimodal deconvolution.

700 Internal exchange standards Pro-Pro-Pro-Ile [PPPI] and Pro-Pro-Pro-Phe [PPPF] were

701 included in each reaction to control for variations in ambient temperature during the labeling 702 reactions (10.1021/ac300535r). Back-exchange was measured by including bradykinin peptide at 
$7032 \mu \mathrm{g} / \mathrm{mL}$ in the deuterated buffer to serve as a fully deuterated control. The back-exchange level

704 ranged from 12-16\% across experiments; deuterium uptake was not corrected.

705 Totally deuterated (TD) samples were prepared by collecting purified peptide eluent following

706 reverse phase LC separation of a pepsin digested undeuterated sample. Following evaporation of

707 the LC elution buffer the peptides were resuspended in HDX PBS pH 7.50 Buffer, deuterated in

708 deuteration Buffer for 1 hours at $65^{\circ} \mathrm{C}$, and quenched and frozen as described above.

709 For peptide identification, un-deuterated peptides were collected from the LC system, dried

710 by speed-vac, and resuspended in 5\% acetonitrie, $0.1 \%$ formic acid for re-injection on an Orbitrap

711 Fusion for MS/MS using EThcD fragmentation. Data was analyzed using Byonic (Protein Metrics)

712 and manually compared to undeuterated sample data.

713

714 Peptic digest analysis:

715 Peptic digest products were collected from the HDX chromatography system, dried by speed-

716 vaccuum, and resuspended in aqueous buffer for nanoscale liquid chromatography (nanoLC-MS)

717 using a 90-minute linear gradient from 2-40\% acetonitrile. Products were analyzed on an Orbitrap

718 Fusion mass spectrometer (ThermoFisher Scientific) using a high-energy collisional dissociation

719 (HCD) product-dependent electron-transfer/high-energy collision dissociation (EThcD) method

720 with a targeted mass list method with a targeted mass list using HexNAc, HexHexNAc, and

721 Hex2HexNAc m/z (204, 366, and 528 respectively) to trigger EThcD.

722 Glycopeptide data were visualized and processed by Byonic ${ }^{\mathrm{TM}}$ (Version 3.8, Protein Metrics Inc.)

723 using a $10 \mathrm{ppm}$ precursor and $10 \mathrm{ppm}$ fragment mass tolerance. Glycopeptides were searched using

724 the N-glycan 309 mammalian database in Protein Metrics PMI-Suite and scored based on the 
725

726

727

728

729

730

731

732

733

734

735

736

737

738

739

740

741

742

743

744

745

746

747

748

749

750

751

assignment of correct c- and z- fragment ions. The true-positive entities were further validated by the presence of glycan oxonium ions m/z at 204 (HexNAc ions) and 366 (HexNAcHex ions).

Supplemental Information: Supplemental information can be found online at ***

\section{References:}

Adhikary, S., D. J. Deredge, A. Nagarajan, L. R. Forrest, P. L. Wintrode and S. K. Singh (2017). "Conformational dynamics of a neurotransmitter:sodium symporter in a lipid bilayer." Proc Natl Acad Sci U S A 114(10): E1786-E1795.

Alfadhli, A., A. O. Staubus, P. R. Tedbury, M. Novikova, E. O. Freed and E. Barklis (2019).

"Analysis of HIV-1 Matrix-Envelope Cytoplasmic Tail Interactions." J Virol 93(21).

Berndsen, Z. T., S. Chakraborty, X. Wang, C. A. Cottrell, J. L. Torres, J. K. Diedrich, C. A. López, J. R. Yates, M. J. van Gils, J. C. Paulson, S. Gnanakaran and A. B. Ward (2020). "Visualization of the HIV-1 Env glycan shield across scales." Proc Natl Acad Sci U S A 117(45): 28014-28025.

Bharat, T. A. and S. H. Scheres (2016). "Resolving macromolecular structures from electron cryo-tomography data using subtomogram averaging in RELION." Nat Protoc 11(11): 20542065.

Cao, L., M. Pauthner, R. Andrabi, K. Rantalainen, Z. Berndsen, J. K. Diedrich, S. Menis, D. Sok, R. Bastidas, S. R. Park, C. M. Delahunty, L. He, J. Guenaga, R. T. Wyatt, W. R. Schief, A. B. Ward, J. R. Yates, D. R. Burton and J. C. Paulson (2018). "Differential processing of HIV envelope glycans on the virus and soluble recombinant trimer." Nat Commun 9(1): 3693.

Carragher, B., N. Kisseberth, D. Kriegman, R. A. Milligan, C. S. Potter, J. Pulokas and A. Reilein (2000). "Leginon: an automated system for acquisition of images from vitreous ice specimens." J Struct Biol 132(1): 33-45. 
Chakrabarti, B. K., L. M. Walker, J. F. Guenaga, A. Ghobbeh, P. Poignard, D. R. Burton and R. 753 T. Wyatt (2011). "Direct antibody access to the HIV-1 membrane-proximal external region 754 positively correlates with neutralization sensitivity." J Virol 85(16): 8217-8226.

755 Checkley, M. A., B. G. Luttge and E. O. Freed (2011). "HIV-1 Envelope Glycoprotein

756 Biosynthesis, Trafficking, and Incorporation." J Mol Biol 410(4): 582-608.

757 Chen, M., J. M. Bell, X. Shi, S. Y. Sun, Z. Wang and S. J. Ludtke (2019). "A complete data 758 processing workflow for cryo-ET and subtomogram averaging." Nat Methods 16(11): 11617591168.

760 Emsley, P., B. Lohkamp, W. G. Scott and K. Cowtan (2010). "Features and development of 761 Coot." Acta Crystallogr D Biol Crystallogr 66(Pt 4): 486-501.

762 Fang, J., K. D. Rand, P. J. Beuning and J. R. Engen (2011). "False EX1 signatures caused by 763 sample carryover during HX MS analyses." Int J Mass Spectrom 302(1-3): 19-25.

764 Frank, G. A., K. Narayan, J. W. Bess, G. Q. Del Prete, X. Wu, A. Moran, L. M. Hartnell, L. A. 765 Earl, J. D. Lifson and S. Subramaniam (2015). "Maturation of the HIV-1 core by a non766 diffusional phase transition." Nat Commun 6: 5854.

767 Frey, G., H. Peng, S. Rits-Volloch, M. Morelli, Y. Cheng and B. Chen (2008). "A fusion768 intermediate state of HIV-1 gp41 targeted by broadly neutralizing antibodies." Proc Natl Acad 769 Sci U S A 105(10): 3739-3744.

770 Fu, Q., M. M. Shaik, Y. Cai, F. Ghantous, A. Piai, H. Peng, S. Rits-Volloch, Z. Liu, S. C. 771 Harrison, M. S. Seaman, B. Chen and J. J. Chou (2018). "Structure of the membrane proximal 772 external region of HIV-1 envelope glycoprotein." Proc Natl Acad Sci U S A 115(38): E8892773 E8899.

774 Gray, E. S., M. C. Madiga, P. L. Moore, K. Mlisana, S. S. Abdool Karim, J. M. Binley, G. M. 775 Shaw, J. R. Mascola and L. Morris (2009). "Broad neutralization of human immunodeficiency 776 virus type 1 mediated by plasma antibodies against the gp41 membrane proximal external 777 region." J Virol 83(21): 11265-11274.

778 Gui, L., J. L. Ebner, A. Mileant, J. A. Williams and K. K. Lee (2016). "Visualization and 779 Sequencing of Membrane Remodeling Leading to Influenza Virus Fusion." J Virol 90(15): 69487806962. 

(2015). "Antibody potency relates to the ability to recognize the closed, pre-fusion form of HIV Env." Nat Commun 6: 6144.

784

Guttman, M., N. K. Garcia, A. Cupo, T. Matsui, J. P. Julien, R. W. Sanders, I. A. Wilson, J. P. Moore and K. K. Lee (2014). "CD4-induced activation in a soluble HIV-1 Env trimer." Structure 22(7): 974-984.

Guttman, M., M. Kahn, N. K. Garcia, S. L. Hu and K. K. Lee (2012). "Solution structure, conformational dynamics, and CD4-induced activation in full-length, glycosylated, monomeric HIV gp120." J Virol 86(16): 8750-8764.

Guttman, M., T. E. Wales, D. Whittington, J. R. Engen, J. M. Brown and K. K. Lee (2016). "Tuning a High Transmission Ion Guide to Prevent Gas-Phase Proton Exchange During H/D Exchange MS Analysis." J Am Soc Mass Spectrom 27(4): 662-668.

Guttman, M., D. D. Weis, J. R. Engen and K. K. Lee (2013). "Analysis of overlapped and noisy hydrogen/deuterium exchange mass spectra." J Am Soc Mass Spectrom 24(12): 1906-1912.

Hamuro, Y. and S. J. Coales (2018). "Optimization of Feasibility Stage for Hydrogen/Deuterium Exchange Mass Spectrometry." J Am Soc Mass Spectrom 29(3): 623-629.

Hill, C. P., D. Worthylake, D. P. Bancroft, A. M. Christensen and W. I. Sundquist (1996). "Crystal structures of the trimeric human immunodeficiency virus type 1 matrix protein: implications for membrane association and assembly." Proc Natl Acad Sci U S A 93(7): 30993104.

Huang, J., B. H. Kang, M. Pancera, J. H. Lee, T. Tong, Y. Feng, H. Imamichi, I. S. Georgiev, G. Y. Chuang, A. Druz, N. A. Doria-Rose, L. Laub, K. Sliepen, M. J. van Gils, A. T. de la Peña, R. Derking, P. J. Klasse, S. A. Migueles, R. T. Bailer, M. Alam, P. Pugach, B. F. Haynes, R. T. Wyatt, R. W. Sanders, J. M. Binley, A. B. Ward, J. R. Mascola, P. D. Kwong and M. Connors (2014). "Broad and potent HIV-1 neutralization by a human antibody that binds the gp41-gp120 interface." Nature 515(7525): 138-142.

Ke, Z., J. Oton, K. Qu, M. Cortese, V. Zila, L. McKeane, T. Nakane, J. Zivanov, C. J. Neufeldt, B. Cerikan, J. M. Lu, J. Peukes, X. Xiong, H. G. Kräusslich, S. H. W. Scheres, R. Bartenschlager and J. A. G. Briggs (2020). "Structures and distributions of SARS-CoV-2 spike proteins on intact virions." Nature 588(7838): 498-502. 

properties of HIV-1 Env without complete neutralization." PLoS Pathog 10(7): e1004271.

Kim, M., Z. Y. Sun, K. D. Rand, X. Shi, L. Song, Y. Cheng, A. F. Fahmy, S. Majumdar, G. Ofek, Y. Yang, P. D. Kwong, J. H. Wang, J. R. Engen, G. Wagner and E. L. Reinherz (2011). "Antibody mechanics on a membrane-bound HIV segment essential for GP41-targeted viral neutralization." Nat Struct Mol Biol 18(11): 1235-1243. T. Bailer, E. M. Cale, L. Chen, C. W. Choi, G. Y. Chuang, N. A. Doria-Rose, A. Druz, I. S. Georgiev, J. Gorman, J. Huang, M. G. Joyce, M. K. Louder, X. Ma, K. McKee, S. O'Dell, M. Pancera, Y. Yang, S. C. Blanchard, W. Mothes, D. R. Burton, W. C. Koff, M. Connors, A. B. Ward, P. D. Kwong and J. R. Mascola (2016). "Fusion peptide of HIV-1 as a site of vulnerability to neutralizing antibody." Science 352(6287): 828-833. dimensional image data using IMOD." J Struct Biol 116(1): 71-76. Guttman, X. Ma, S. Narpala, C. Soto, D. S. Terry, Y. Yang, T. Zhou, G. Ahlsen, R. T. Bailer, M. Chambers, G. Y. Chuang, N. A. Doria-Rose, A. Druz, M. A. Hallen, A. Harned, T. Kirys, M. K. Louder, S. O'Dell, G. Ofek, K. Osawa, M. Prabhakaran, M. Sastry, G. B. Stewart-Jones, J. Stuckey, P. V. Thomas, T. Tittley, C. Williams, B. Zhang, H. Zhao, Z. Zhou, B. R. Donald, L. K. Lee, S. Zolla-Pazner, U. Baxa, A. Schon, E. Freire, L. Shapiro, K. K. Lee, J. Arthos, J. B. Munro, S. C. Blanchard, W. Mothes, J. M. Binley, A. B. McDermott, J. R. Mascola and P. D. Kwong (2015). "Crystal structure, conformational fixation and entry-related interactions of mature ligand-free HIV-1 Env." Nat Struct Mol Biol 22(7): 522-531. envelope spikes through directed evolution." PLoS Pathog 9(2): e1003184.

Lee, J. H., D. P. Leaman, A. S. Kim, A. Torrents de la Pena, K. Sliepen, A. Yasmeen, R. Derking, A. Ramos, S. W. de Taeye, G. Ozorowski, F. Klein, D. R. Burton, M. C. Nussenzweig, P. Poignard, J. P. Moore, P. J. Klasse, R. W. Sanders, M. B. Zwick, I. A. Wilson and A. B. Ward (2015). "Antibodies to a conformational epitope on gp41 neutralize HIV-1 by destabilizing the 840 Env spike." Nat Commun 6: 8167. 
Li, Z., W. Li, M. Lu, J. Bess, C. W. Chao, J. Gorman, D. S. Terry, B. Zhang, T. Zhou, S. C. Blanchard, P. D. Kwong, J. D. Lifson, W. Mothes and J. Liu (2020). "Subnanometer structures of HIV-1 envelope trimers on aldrithiol-2-inactivated virus particles." Nat Struct Mol Biol 27(8): 726-734.

Madeira, F., Y. M. Park, J. Lee, N. Buso, T. Gur, N. Madhusoodanan, P. Basutkar, A. R. N. Tivey, S. C. Potter, R. D. Finn and R. Lopez (2019). "The EMBL-EBI search and sequence analysis tools APIs in 2019." Nucleic Acids Res 47(W1): W636-W641.

Majumdar, R., P. Manikwar, J. M. Hickey, J. Arora, C. R. Middaugh, D. B. Volkin and D. D. Weis (2012). "Minimizing carry-over in an online pepsin digestion system used for the H/D exchange mass spectrometric analysis of an IgG1 monoclonal antibody." J Am Soc Mass Spectrom 23(12): 2140-2148.

Mastronarde, D. N. (2005). "Automated electron microscope tomography using robust prediction of specimen movements." J Struct Biol 152(1): 36-51.

Mastronarde, D. N. and S. R. Held (2017). "Automated tilt series alignment and tomographic reconstruction in IMOD." J Struct Biol 197(2): 102-113.

McGuire, A. T., S. Hoot, A. M. Dreyer, A. Lippy, A. Stuart, K. W. Cohen, J. Jardine, S. Menis, J. F. Scheid, A. P. West, W. R. Schief and L. Stamatatos (2013). "Engineering HIV envelope protein to activate germline B cell receptors of broadly neutralizing anti-CD4 binding site antibodies." J Exp Med 210(4): 655-663.

Nicastro, D., C. Schwartz, J. Pierson, R. Gaudette, M. E. Porter and J. R. McIntosh (2006). "The molecular architecture of axonemes revealed by cryoelectron tomography." Science 313(5789): 944-948.

Pan, J., H. Peng, B. Chen and S. C. Harrison (2020). "Cryo-EM Structure of Full-length HIV-1 Env Bound With the Fab of Antibody PG16." J Mol Biol 432(4): 1158-1168.

Pandey, A. and A. P. Galvani (2019). "The global burden of HIV and prospects for control." Lancet HIV 6(12): e809-e811.

Pettersen, E. F., T. D. Goddard, C. C. Huang, G. S. Couch, D. M. Greenblatt, E. C. Meng and T. E. Ferrin (2004). "UCSF Chimera--a visualization system for exploratory research and analysis." J Comput Chem 25(13): 1605-1612. 
872

873

874

875

876

877

878

879

880

881

882

883

884

885

886

887

888

889

890

891

892

893

894

895

896

897

898

899

900

901

902

Pettersen, E. F., T. D. Goddard, C. C. Huang, E. C. Meng, G. S. Couch, T. I. Croll, J. H. Morris and T. E. Ferrin (2021). "UCSF ChimeraX: Structure visualization for researchers, educators, and developers." Protein Sci 30(1): 70-82.

Piai, A., Q. Fu, Y. Cai, F. Ghantous, T. Xiao, M. M. Shaik, H. Peng, S. Rits-Volloch, W. Chen, M. S. Seaman, B. Chen and J. J. Chou (2020). "Structural basis of transmembrane coupling of the HIV-1 envelope glycoprotein." Nat Commun 11(1): 2317.

Rantalainen, K., Z. T. Berndsen, A. Antanasijevic, T. Schiffner, X. Zhang, W. H. Lee, J. L. Torres, L. Zhang, A. Irimia, J. Copps, K. H. Zhou, Y. D. Kwon, W. H. Law, C. A. Schramm, R. Verardi, S. J. Krebs, P. D. Kwong, N. A. Doria-Rose, I. A. Wilson, M. B. Zwick, J. R. Yates, W. R. Schief and A. B. Ward (2020). "HIV-1 Envelope and MPER Antibody Structures in Lipid Assemblies." Cell Rep 31(4): 107583.

Rawi, R., L. Rutten, Y. T. Lai, A. S. Olia, S. Blokland, J. Juraszek, C. H. Shen, Y. Tsybovsky, R. Verardi, Y. Yang, B. Zhang, T. Zhou, G. Y. Chuang, P. D. Kwong and J. P. M. Langedijk (2020). "Automated Design by Structure-Based Stabilization and Consensus Repair to Achieve Prefusion-Closed Envelope Trimers in a Wide Variety of HIV Strains." Cell Rep 33(8): 108432.

Rossio, J. L., M. T. Esser, K. Suryanarayana, D. K. Schneider, J. W. Bess, G. M. Vasquez, T. A. Wiltrout, E. Chertova, M. K. Grimes, Q. Sattentau, L. O. Arthur, L. E. Henderson and J. D. Lifson (1998). "Inactivation of human immunodeficiency virus type 1 infectivity with preservation of conformational and functional integrity of virion surface proteins." J Virol 72(10): 7992-8001.

Roy, N. H., J. Chan, M. Lambelé and M. Thali (2013). "Clustering and mobility of HIV-1 Env at viral assembly sites predict its propensity to induce cell-cell fusion." J Virol 87(13): 7516-7525.

Scheres, S. H. (2012). "RELION: implementation of a Bayesian approach to cryo-EM structure determination." J Struct Biol 180(3): 519-530.

Schneider, C. A., W. S. Rasband and K. W. Eliceiri (2012). "NIH Image to ImageJ: 25 years of image analysis." Nat Methods 9(7): 671-675.

Schur, F. K., W. J. Hagen, M. Rumlová, T. Ruml, B. Müller, H. G. Kräusslich and J. A. Briggs (2015). "Structure of the immature HIV-1 capsid in intact virus particles at $8.8 \AA$ resolution." Nature 517(7535): 505-508.

Stadtmueller, B. M., M. D. Bridges, K. M. Dam, M. T. Lerch, K. E. Huey-Tubman, W. L. Hubbell and P. J. Bjorkman (2018). "DEER Spectroscopy Measurements Reveal Multiple 
Conformations of HIV-1 SOSIP Envelopes that Show Similarities with Envelopes on Native Virions." Immunity 49(2): 235-246.e234. Wyatt, A. J. Olson and M. B. Zwick (2017). "Dense Array of Spikes on HIV-1 Virion Particles."

907 J Virol 91(14). Wagh, T. Zhou, A. J. Behrens, T. Bylund, C. W. Choi, J. R. Davison, I. S. Georgiev, M. G. Joyce, Y. D. Kwon, M. Pancera, J. Taft, Y. Yang, B. Zhang, S. S. Shivatare, V. S. Shivatare, C. C. Lee, C. Y. Wu, C. A. Bewley, D. R. Burton, W. C. Koff, M. Connors, M. Crispin, U. Baxa, B. T. Korber, C. H. Wong, J. R. Mascola and P. D. Kwong (2016). "Trimeric HIV-1-Env Structures Define Glycan Shields from Clades A, B, and G." Cell 165(4): 813-826.

914 Tedbury, P. R. and E. O. Freed (2014). "The role of matrix in HIV-1 envelope glycoprotein 915 incorporation." Trends Microbiol 22(7): 372-378.

916 Tedbury, P. R., M. Novikova, S. D. Ablan and E. O. Freed (2016). "Biochemical evidence of a 917 role for matrix trimerization in HIV-1 envelope glycoprotein incorporation." Proc Natl Acad Sci 918 U S A 113(2): E182-190. Tong, T., E. T. Crooks, K. Osawa and J. M. Binley (2012). "HIV-1 virus-like particles bearing 920 pure env trimers expose neutralizing epitopes but occlude nonneutralizing epitopes." J Virol 921 86(7): 3574-3587.

922 van Gils, M. J. and R. W. Sanders (2013). "Broadly neutralizing antibodies against HIV-1: 923 templates for a vaccine." Virology 435(1): 46-56.

924 Verkerke, H. P., J. A. Williams, M. Guttman, C. A. Simonich, Y. Liang, M. Filipavicius, S. L. $925 \mathrm{Hu}, \mathrm{J}$. Overbaugh and K. K. Lee (2016). "Epitope-Independent Purification of Native-Like 926 Envelope Trimers from Diverse HIV-1 Isolates." J Virol 90(20): 9471-9482.

927 Wang, Y., P. Kaur, Z. J. Sun, M. A. Elbahnasawy, Z. Hayati, Z. S. Qiao, N. N. Bui, C. Chile, M. 928 L. Nasr, G. Wagner, J. H. Wang, L. Song, E. L. Reinherz and M. Kim (2019). "Topological 929 analysis of the gp41 MPER on lipid bilayers relevant to the metastable HIV-1 envelope prefusion 930 state." Proc Natl Acad Sci U S A 116(45): 22556-22566.

931 Ward, A. B. and I. A. Wilson (2015). "Insights into the trimeric HIV-1 envelope glycoprotein 932 structure." Trends Biochem Sci 40(2): 101-107. 
933 Wei, X., J. M. Decker, S. Wang, H. Hui, J. C. Kappes, X. Wu, J. F. Salazar-Gonzalez, M. G. 934 Salazar, J. M. Kilby, M. S. Saag, N. L. Komarova, M. A. Nowak, B. H. Hahn, P. D. Kwong and 935 G. M. Shaw (2003). "Antibody neutralization and escape by HIV-1." Nature 422(6929): 307936312.

937 Wyma, D. J., J. Jiang, J. Shi, J. Zhou, J. E. Lineberger, M. D. Miller and C. Aiken (2004). 938 "Coupling of human immunodeficiency virus type 1 fusion to virion maturation: a novel role of 939 the gp41 cytoplasmic tail." J Virol 78(7): 3429-3435.

940 Xiong, Q., M. K. Morphew, C. L. Schwartz, A. H. Hoenger and D. N. Mastronarde (2009). "CTF 941 determination and correction for low dose tomographic tilt series." J Struct Biol 168(3): 378-387.

942 Zheng, S. Q., E. Palovcak, J. P. Armache, K. A. Verba, Y. Cheng and D. A. Agard (2017).

943 "MotionCor2: anisotropic correction of beam-induced motion for improved cryo-electron 944 microscopy." Nat Methods 14(4): 331-332. 